\title{
Radioactivité d'origine naturelle dans l'environnement en France : niveaux non perturbés par l'homme
}

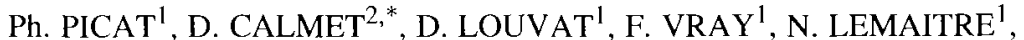 \\ G. LINDEN $^{1}$, P. BARBEY ${ }^{3}$, G. PIGREE $^{3}$, T. BOURCIER ${ }^{4}$, F. LEVY ${ }^{5}$, S. LE BAR $^{5}$, \\ Y. BARON ${ }^{6}$, D. DELACROIX ${ }^{2}$, E. PANAÏVA ${ }^{2}$, T. DE BRUYNE ${ }^{2}$, J.Y. HERVÉ ${ }^{2}$,

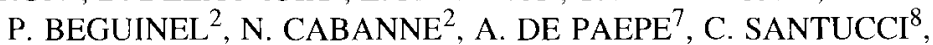 \\ T. PROT $^{8}$, J.L. MATRAY ${ }^{9}$, J.L. TILLIE ${ }^{10}$
}

(Manuscrit reçu le 10 avril 2002, accepté le 3 juin 2002)

RÉSUMÉ Quelles sont les valeurs qui caractérisent sur le territoire français les rayonnements ionisants et les radionucléides d'origine naturelle ? Comment distinguer dans le résultat d'une mesure la part qui résulte éventuellement d'une activité humaine ? Afin d'essayer de répondre à ces questions, un questionnaire a été adressé aux différents organismes qui effectuent en France des mesures de radioactivité dans l'environnement. Les informations mentionnées dans cette étude se rapportent exclusivement à des observations faites dans l'environnement à l'extérieur des bâtiments et du périmètre des installations nucléaires de base (INB). En outre, elles ne concernent, sauf cas particuliers signalés, que des mesures de rayonnements ou de radioéléments d'origine naturelle dont les résultats n'ont pas été perturbés par l'action de l'homme. L'enquête montre la difficulté actuelle de dresser en France un bilan exhaustif des connaissances acquises concernant les caractéristiques du bruit de fond radioactif d'origine naturelle dans l'environnement. Aujourd'hui en France, dans le domaine de la radioactivité naturelle, les efforts consentis manquent de coordination et d'orientation vers certaines priorités. À ce titre, les mesures des ${ }^{238} \mathrm{U}$ et ${ }^{232}$ Th précurseurs des ${ }^{226} \mathrm{Ra}$ et ${ }^{228} \mathrm{Ra}$, des descendants solides émetteurs alpha du radon (par la mesure des EAP du ${ }^{222} \mathrm{Rn}$ et ${ }^{220} \mathrm{Rn}$ ), des émetteurs gamma post émanation $\left({ }^{214} \mathrm{~Pb},{ }^{214} \mathrm{Bi},{ }^{212} \mathrm{~Pb}\right), \mathrm{du}{ }^{210} \mathrm{~Pb},{ }^{210} \mathrm{Po}$ et du ${ }^{14} \mathrm{C}$ doivent être privilégiées.

Abstract Natural radioactivity in the environment of France: levels free from human interference.

On the french territory which levels are characteristic of ionising radiation and which of radionuclides natural in origin? Is it possible to distinguish the portion of

\footnotetext{
* Auteur pour correspondance : CEADAM/DMSE, BP 12, 91680 Bruyères-le-Châtel, France.

IRSN, France.

CEA, France.

Association pour le contrôle de la radioactivité dans l'ouest, France.

EDF, France.

COGEMA, France.

Marine nationale, France

Centre d'essais et de contrôle de la radioactivité, France.

${ }^{8}$ Agence nationale pour la gestion des déchets radioactifs, France.

${ }^{9}$ Bureau de recherches gćologiques et minières, France.

${ }^{10}$ Direction générale de la concurrence, consommation et répression des fraudes, France.
} 


\begin{abstract}
a measurement readily attributable to man-made sources? In an attempt to answer these questions, a questionnaire was sent to the various bodies measuring environmental radioactivity in France. Information contained in this paper relates solely to observation of the environment (open spaces) outside buildings and beyond the perimetre of nuclear facilities. Further more, it concerns the measurement of radiation or of radionuclides natural in origin where the results have not been influenced by human activity, with the exception of specifically mentioned cases. The survey illustrates the present difficulty of assessing the acquired data on the characteristics of natural radioactive background noise in the environment in France. Efforts exerted in France in the area of natural radioactivity lack coordination and a shared setting of priorities. In this regard, the measurement of ${ }^{238} \mathrm{U}$ and ${ }^{232} \mathrm{Th}$ precursors to ${ }^{226} \mathrm{Ra}$ and ${ }^{228} \mathrm{Ra}$ of solid alpha-emitting radon daughters (by measuring PAE of ${ }^{222} \mathrm{Rn}$ and ${ }^{220} \mathrm{Rn}$ ), of post-emanation gamma emitters $\left({ }^{214} \mathrm{~Pb},{ }^{214} \mathrm{Bi},{ }^{212} \mathrm{~Pb}\right)$ and of ${ }^{210} \mathrm{~Pb},{ }^{210} \mathrm{Po}$ and ${ }^{11} \mathrm{C}$ should be given top priority.
\end{abstract}

\title{
1. Introduction
}

Dans le monde, seules quelques zones habituellement proches de sites où se sont produits des accidents nucléaires majeurs, comme Tchernobyl en Ukraine et Kyshtym en Russie, se caractérisent par des niveaux d'activité des radioéléments d'origine artificielle présents dans l'environnement plus importants que ceux dus aux radioéléments d'origine naturelle.

En tout autre lieu de la planète, l'exposition aux rayonnements ionisants des populations résulte principalement des irradiations externes et internes liées à des sources de radioactivités naturelles (Jammet, 1984 ; UNSCEAR, 1988, 1993 ; Thébault et al., 1998). Des différences notables apparaissent toutefois au niveau des isotopes radioactifs naturels détectés et de leurs concentrations selon les caractéristiques géologiques, orographiques et biologiques de la région, de l'écosystème ou du constituant de la biosphère considéré. Enfin, l'existence d'installations, en fonctionnement ou à l'arrêt, qui produisent ou détiennent des matières radioactives d'origine naturelle, peut également localement modifier les niveaux d'activité de ces isotopes ou éléments naturels.

Quelles sont donc les valeurs qui caractérisent les rayonnements ionisants et les radionucléides d'origine naturelle sur le territoire français ? Comment distinguer dans le résultat d'une mesure la part qui résulte éventuellement d'une activité humaine ? L'impact dosimétrique de cette dernière doit en effet être comptabilisé dans la limite de dose efficace de $1000 \mu \mathrm{Sv}$ par an qui s'applique depuis le 13 mai 2000 aux personnes du public dans tout État membre de la Communauté européenne (JOCE, 1996).

Afin d'essayer de répondre à l'ensemble de ces questions, un questionnaire a été adressé aux différents organismes qui effectuent en France des mesures de 
radioactivité dans l'environnement, qu'ils interviennent dans un but de contrôle, y compris d'auto-contrôle, de recherche ou d'expertise, en tant qu'exploitants, services de l'État, entreprises privées ou associations (Tab. I)

Les informations mentionnées dans cette étude se rapportent exclusivement à des observations faites dans l'environnement à l'extérieur des bâtiments et du périmètre des installations nucléaires de base (INB). En outre, elles ne concernent, sauf cas particuliers signalés, que des mesures de rayonnements ou de radioéléments d'origine naturelle dont les résultats n'ont pas été perturbés par l'action de l'homme.

\section{Composantes du bruit de fond radioactif naturel et causes de perturbation}

Dans la biosphère, l'exposition des êtres vivants aux radiations ionisantes issue de sources naturelles résulte de l'existence de rayonnements et de radionucléides d'origine cosmique et tellurique.

\subsection{Rayonnement cosmique}

Il a pour origine le bombardement continu de la Terre par des particules chargées de hautes énergies issues de l'espace intersidéral. Ces particules cosmiques primaires interagissent avec les constituants de l'atmosphère terrestre et donnent naissance au niveau du sol à un rayonnement composé de $80 \%$ de muons et $20 \%$ d'électrons. L'intensité de ce rayonnement varie principalement en fonction de l'altitude, de la latitude et du cycle de 11 ans de l'activité solaire.

Au niveau de la mer et à une latitude de $45^{\circ}$, l'irradiation externe ${ }^{11}$ associée aux particules chargées du rayonnement cosmique, est de $31 \mathrm{nGy} / \mathrm{h}$ et représente pour un individu qui séjourne en moyenne $20 \%$ de son temps à l'extérieur des bâtiments, une dose annuelle ${ }^{11}$ due aux muons de $54 \mu \mathrm{Sv}$, celle due aux neutrons étant de $16 \mu \mathrm{Sv}$.

À titre de comparaison, les doses annuelles des personnes empruntant régulièrement l'avion et des personnels navigants peuvent atteindre $5000 \mu \mathrm{Sv}$ par an.

\subsection{Radioéléments naturels d'origine cosmique}

Les réactions des particules cosmiques primaires avec l'azote, l'oxygène et l'argon présents dans les couches supérieures de la stratosphère donnent aussi

\footnotetext{
${ }^{\prime}$ Ces valeurs citées dans ce paragraphe sont extraites de rapports (UNSCEAR, 1988, 1993) el correspondent à des moyennes mondiales pour des adultes.
} 


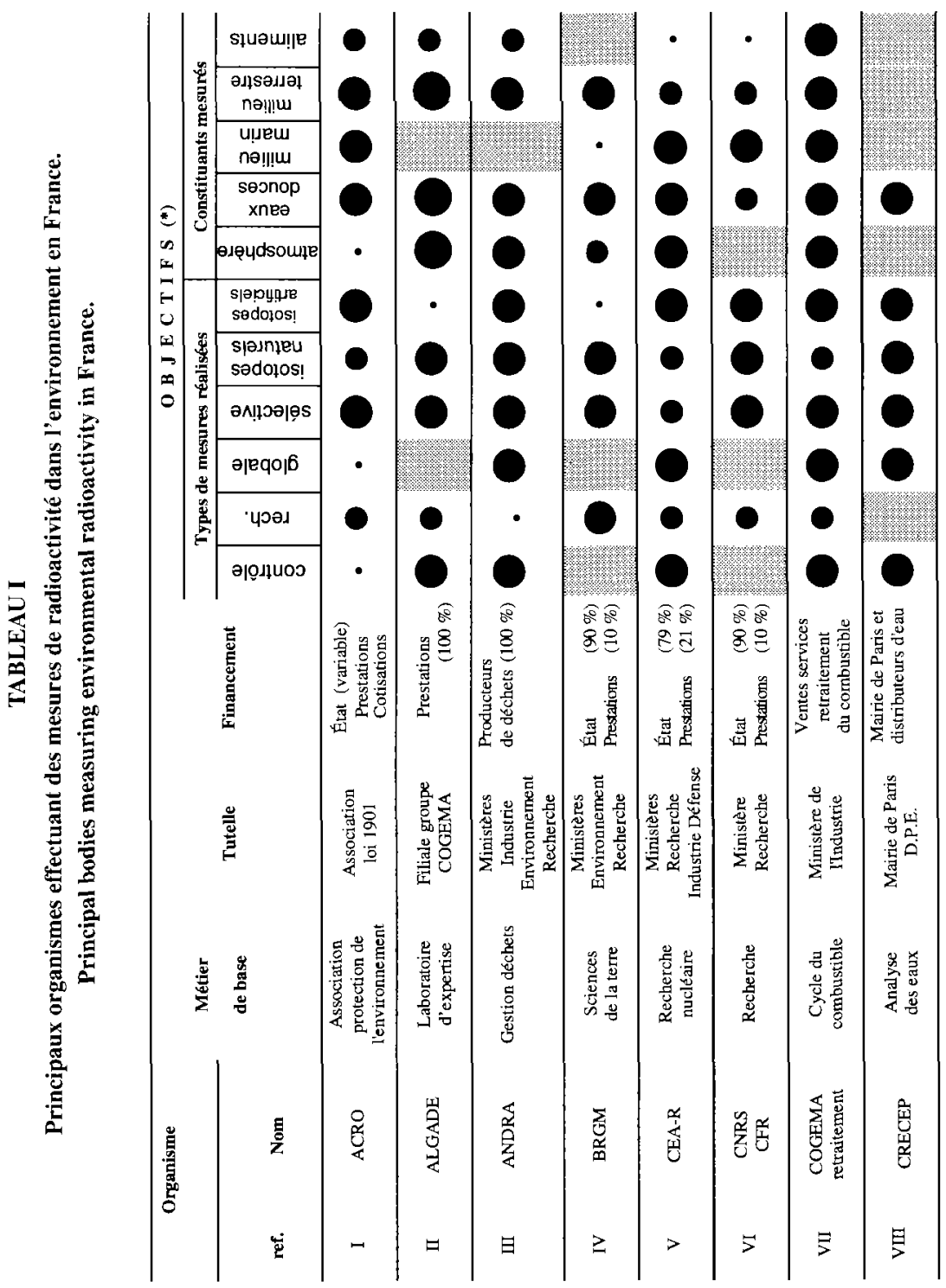




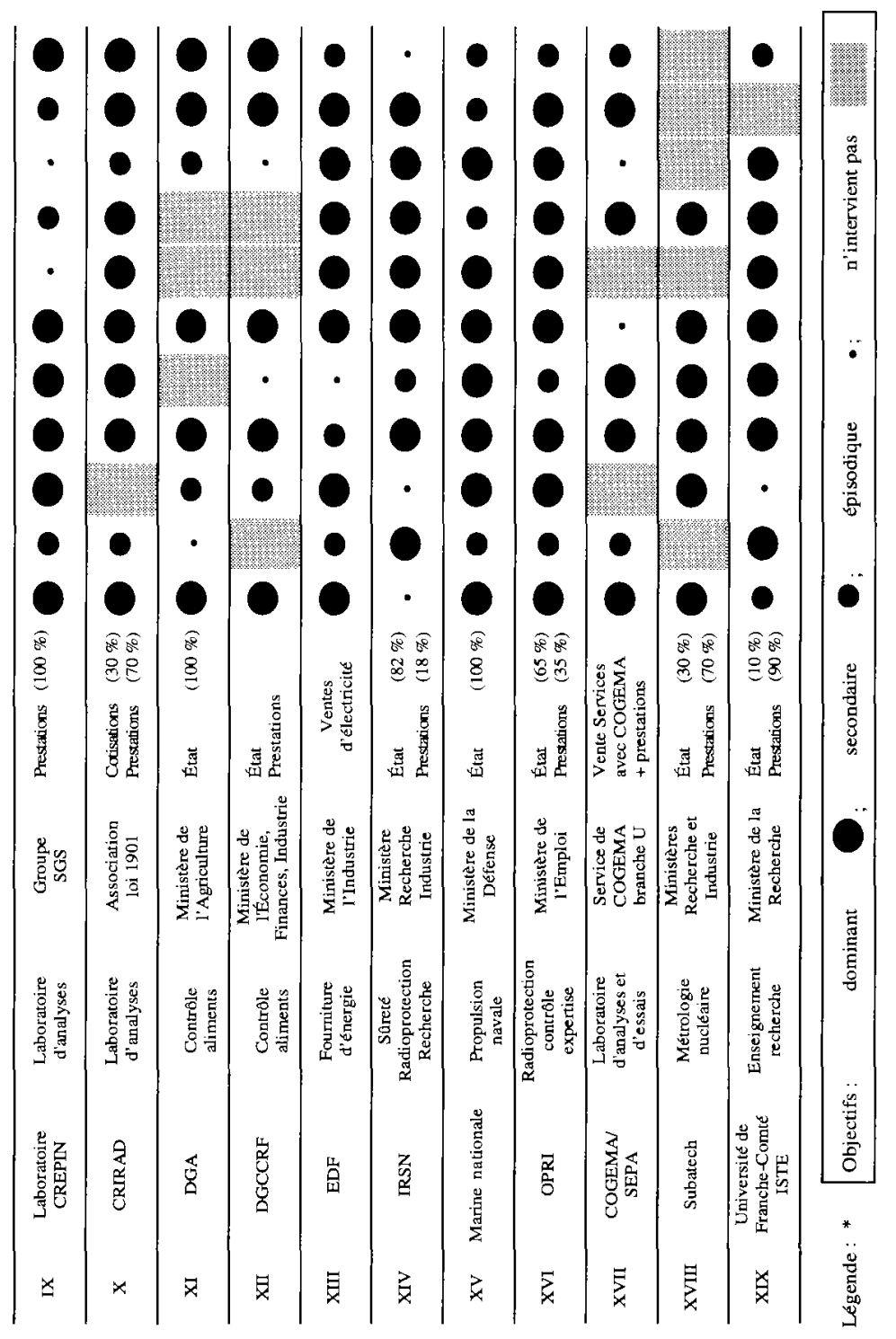


naissance à une série de radionucléides cosmogéniques tels que le tritium $\left({ }^{3} \mathrm{H}\right)$, le béryllium-7 $\left({ }^{7} \mathrm{Be}\right)$, le carbone- $14\left({ }^{14} \mathrm{C}\right)$ et le sodium-22 $\left({ }^{22} \mathrm{Na}\right)$ dont les principales caractéristiques sont présentées au tableau II.

Le taux de production de ces radioéléments varie aussi en fonction de l'altitude, de la latitude et du cycle solaire (Calmet et al., 1998a). Au niveau du sol, leur abondance et leur répartition dans la biosphère dépendent par ailleurs de nombreux phénomènes :

- échanges entre la stratosphère et la troposphère,

- dépôt et captation des formes gazeuses et particulaires (aérosols) sur les surfaces et le couvert végétal,

- incorporation de certains isotopes $\left({ }^{3} \mathrm{H},{ }^{14} \mathrm{C}\right)$ dans la biomasse lors de la photosynthèse.

L'intensité de ces mécanismes est liée à l'état physiologique des plantes et varie également selon la saison, le lieu et le moment de la journée d'où de grandes fluctuations susceptibles d'être observées dans l'environnement (Belot et al., 1996).

La dose annuelle due aux radionucléides cosmogéniques provient essentiellement du ${ }^{14} \mathrm{C}$ et représente $12 \mu \mathrm{Sv}$. Les contributions dues aux ${ }^{22} \mathrm{Na},{ }^{7} \mathrm{Be}$ et ${ }^{3} \mathrm{H}$ sont beaucoup plus modestes et atteignent respectivement $0,15,0,03$ et $0,01 \mu \mathrm{Sv}$.

Il faut toutefois noter que certains isotopes comme le tritium et le carbone-14 peuvent être aussi produits de façon artificielle (Caput et al., 1981). Par exemple, dans les centrales électronucléaires françaises, à la production de $1 \mathrm{kWh}$ est associé un rejet en tritium sous forme liquide de 1,7 kBq (OPRI, 1997). En 1997, l'ensemble du parc français a ainsi généré $0,67 \mathrm{PBq}$ de tritium ce qui équivaut à environ $1 \%$ de sa production naturelle annuelle mondiale (Tab. II). D'autres sources artificielles de tritium plus anciennes existent, telles celles liées aux anciennes explosions aériennes d'engins nucléaires qui aujourd'hui ont une importance moindre. Enfin, les usines de retraitement du combustible constituent une source industrielle importante de tritium, le seul site de La Hague en ayant rejeté, en 1995, de l'ordre de 9,6 PBq dans la Manche et $0,085 \mathrm{PBq}$ dans l'atmosphère.

Aussi, les concentrations en tritium en de nombreuses régions ne peuvent être considérées comme imputables au seul bruit de fond d'origine naturelle qui dans les eaux représente une activité qui varie entre 0,12 à $0,24 \mathrm{~Bq}^{-1}$ (Tort et al., 1997).

\subsection{Radioéléments naturels d'origine tellurique}

Aujourd'hui ne subsistent en raison de leur période radioactive, qu'une vingtaine de radionucléides présents au moment de la formation de la Terre. Certains comme 


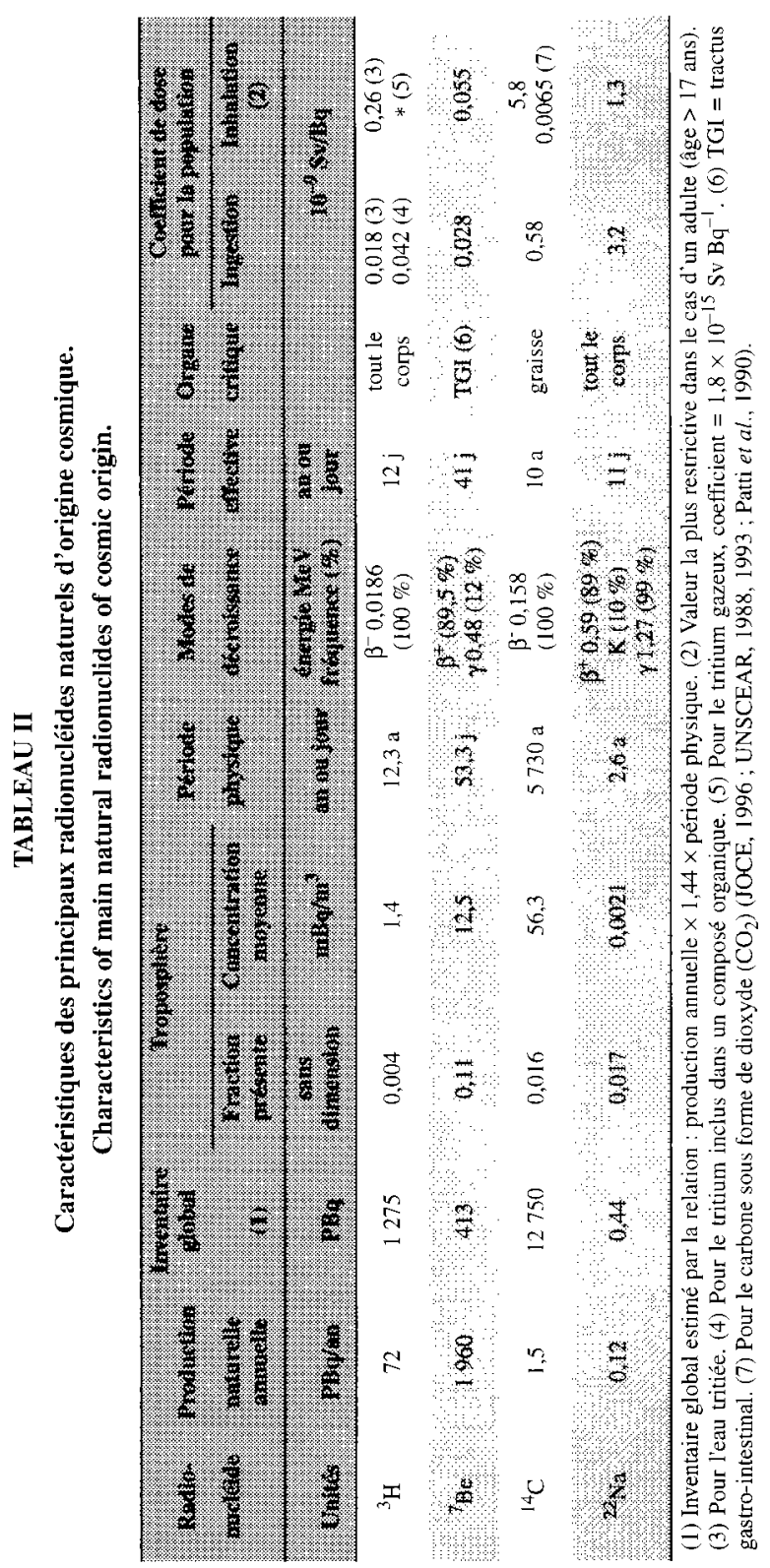


le potassium-40 $\left({ }^{40} \mathrm{~K}\right)$ se désintègrent en donnant directement un élément stable. D'autres, au contraire, comme l'uranium-238 $\left({ }^{238} \mathrm{U}\right)$, le thorium-232 $\left({ }^{232} \mathrm{Th}\right)$ et l'uranium-235 $\left({ }^{235} \mathrm{U}\right)$ donnent naissance à des séries de descendants radioactifs de propriétés chimiques et physiques distinctes de celles des nucléides pères (SFRP, 1998). L'ensemble de ces filiations constitue les familles radioactives naturelles. Les caractéristiques des principaux radioéléments naturels d'origine tellurique sont présentées au tableau III.

Leur comportement dans la biosphère est conditionné par les propriétés des éléments chimiques auxquels se rattache chaque isotope mais aussi par les caractéristiques des eaux, des sols, de la flore et de la faune (AIEA, 1994). Par exemple, l'existence de formes gazeuses et solubles anioniques pour un même isotope, est favorable à sa migration dans l'environnement. A contrario, des formes particulaires ou solubles cationiques conduisent en général à une mobilité plus réduite. Il en résulte des variations importantes des concentrations des isotopes des familles de l'uranium et du thorium dans les différents compartiments de la biosphère.

En outre, les possibilités de modification par l'homme du bruit de fond radioactif naturel d'origine tellurique sont multiples (EC, 1998), notamment à proximité des sites d'extraction et de traitement des minerais d'uranium ou de thorium, des anciennes usines d'utilisation du radium-226 (peinture, horlogerie, médecine), mais aussi au voisinage des usines de production d'engrais phosphatés ou des sites d'extraction de gaz et de pétrole (Aigueperse et al., 1981 ; Descamps et Baudin-Jaulent, 1987 ; Zettwoog et al., 1997).

La dose annuelle due au ${ }^{40} \mathrm{~K}$ est limitée à $170 \mu \mathrm{Sv}{ }^{12}$. Une valeur identique est atteinte pour les isotopes de période longue des familles de ${ }^{238} \mathrm{U}$ et du ${ }^{232} \mathrm{Th}$.

Les doses annuelles dues à l'inhalation des isotopes gazeux du radon $\left({ }^{222} \mathrm{Rn}\right.$ et ${ }^{220} \mathrm{Rn}$ ) sont évaluées séparément car elles résultent de modalités particulières de transfert dans l'environnement (ICRP, 1993). Leur impact chez l'homme est en effet lié à l'existence de descendants solides émetteurs alpha à vie courte $\left({ }^{218} \mathrm{Po}\right.$, ${ }^{214} \mathrm{Po}$ et ${ }^{212} \mathrm{Bi}$ ) qui se déposent dans les voies respiratoires. Les doses annuelles dues à l'inhalation des ${ }^{222} \mathrm{Rn},{ }^{220} \mathrm{Rn}$ et à leurs descendants sont estimées respectivement à $60^{13}$ et $10 \mu \mathrm{Sv}$.

\footnotetext{
${ }^{12}$ Compte tenu de son contrôle homéostatique dans le corps humain, où sa concentration est de l'ordre de $0,18 \%$.

${ }^{13}$ Pour un individu séjournant $20 \%$ de son temps à l'extérieur des bâtiments, une concentration moyenne en ${ }^{222}$ Rn dans l'air de $10 \mathrm{~Bq} \mathrm{~m}{ }^{-3}$ et un facteur d'équilibre de 0,6 entre lc ${ }^{222} \mathrm{R}_{n}$ et ses descendants à vie courte.
} 


\section{TABLEAU III}

Caractéristiques des principaux radionucléides naturels d'origine tellurique.

Characteristics of main natural radionuclides of telluric origin.

\begin{tabular}{|c|c|c|c|c|c|c|c|c|}
\hline \multirow{3}{*}{ 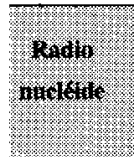 } & \multirow{3}{*}{ 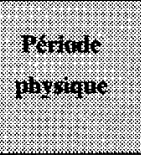 } & \multirow{3}{*}{\multicolumn{2}{|c|}{ 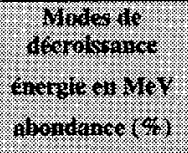 }} & \multirow{3}{*}{ 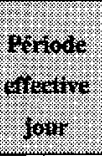 } & \multirow{3}{*}{ 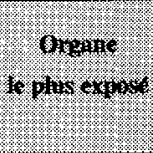 } & \multicolumn{2}{|c|}{ 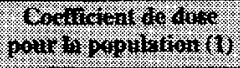 } & \multirow{3}{*}{ 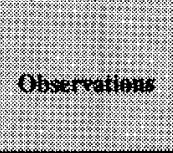 } \\
\hline & & & & & & ngestoh & how $10 \%$ & \\
\hline & & & & & & \multicolumn{2}{|c|}{$\left(p^{2}\right)=0 \%$} & \\
\hline${ }^{40} \mathrm{~K}$ & $1,28 \times 10^{10} a$ & $\begin{array}{l}\beta 1,32 \\
\gamma 1,46\end{array}$ & $\begin{array}{l}(89) \\
(11)\end{array}$ & & & 6,2 & 2,1 & $\begin{array}{l}\text { Régulation } \\
\text { homéostatique } \\
\text { che } z \text { l'homme }\end{array}$ \\
\hline 2380 & $4,5 \times 10^{\circ} \mathrm{a}$ & $\begin{array}{l}\times 4,19 \\
19,48\end{array}$ & $(200)$ & 100 & $\mathrm{TGI}$ & 45 & 800 & 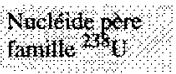 \\
\hline${ }^{234} \mathrm{U}$ & $2,5 \times 10^{5} a$ & $\begin{array}{l}\alpha 4,77 \\
4,72\end{array}$ & $\begin{array}{l}(72) \\
(28)\end{array}$ & 100 & TGI & 49 & 9400 & \\
\hline $270 \mathrm{~K}$ & मे $\times 10^{4}$ & 4,61 & $(24)$ & 70000 & os & 210 & 100000 & \\
\hline${ }^{226} \mathrm{Ra}$ & $1,6 \times 10^{3} \mathrm{a}$ & $\begin{array}{l}\alpha 4,78 \\
4,59\end{array}$ & $\begin{array}{r}(95) \\
(5)\end{array}$ & 16000 & os & 280 & 9500 & \\
\hline $22 \mathrm{Ra}$ & 3,83 & $\alpha, 5,9$ & 100 & (2) & pounnos & & (2) & Gat odiogetif \\
\hline${ }^{218} \mathrm{Po}$ & $3,05 \mathrm{~min}$ & $\alpha 6,0$ & (99) & & & & & \\
\hline${ }^{2] 4} \mathrm{~Pb}$ & $0,447 \mathrm{~h}$ & $\beta-1,03$ & $(6)$ & & & 0,14 & 15 & Descendants \\
\hline${ }^{214} \mathrm{Bi}$ & $0,332 \mathbf{h}$ & $\begin{array}{l}\beta 3,18 \\
\gamma 0,61\end{array}$ & (99) & & poumons & 0,11 & 14 & $\begin{array}{l}\text { solides à vie } \\
\text { courte du }{ }^{222} \mathbf{R n}\end{array}$ \\
\hline${ }^{214} \mathrm{PO}_{0}$ & $160 \mu \mathrm{s}$ & $\alpha 7,68$ & $(100)$ & & & & & \\
\hline $210 \mathrm{pb}$ & 22,3 & 60,04 & $(8)$ & 1200 & compentiet & 690 & 5600 & \\
\hline${ }^{210} \mathrm{Po}$ & $138,4 \mathrm{j}$ & $\alpha 5,305$ & $(100)$ & 45 & rate, reins & 1200 & 4300 & \\
\hline मै। & $7,1 \times \rho^{2}$ ' & $\begin{array}{l}0,35 \\
4,31 \\
4,56 \\
0,189\end{array}$ & $\begin{array}{l}(35) \\
(6) \\
65)\end{array}$ & 100 & TGI & 47 & 8500 & \\
\hline${ }^{232} \mathrm{Th}$ & $1,4 \times 10^{10} \mathrm{a}$ & $\begin{array}{l}\alpha 4,01 \\
4,61 \\
\gamma 0,059\end{array}$ & $\begin{array}{l}(76) \\
(24) \\
(24)\end{array}$ & 70000 & os & 230 & 110000 & \\
\hline $28 \mathrm{Ra}$ & 67 & 1002 & 100 & 2120 & as & 690 & 16000 & \\
\hline${ }^{224} \mathrm{Ra}$ & $3,64 \mathbf{j}$ & $\begin{array}{l}\alpha 5,68 \\
5,44 \\
\gamma 0,241\end{array}$ & $\begin{array}{r}(95) \\
(5) \\
(5)\end{array}$ & 3,6 & os & 65 & 3400 & \\
\hline $22 \% \mathrm{RH}$ & $s \mathrm{~s}$ & 06,28 & 09 & (2) & poumous & & & Gaz radioacti \\
\hline${ }^{216} \mathrm{Po}$ & $0,16 \mathrm{~s}$ & $\alpha 6,78$ & & & poumons & & & \\
\hline${ }^{212} \mathrm{~Pb}$ & $10,6 \mathrm{~h}$ & $\begin{array}{l}\beta^{-} 0,34 \\
0,58\end{array}$ & $\begin{array}{l}(84) \\
(12)\end{array}$ & 0,44 & TGI, reins & 6,0 & 190 & $\begin{array}{l}\text { Descendants } \\
\text { solides à vie } \\
\text { courte du }{ }^{220} \mathrm{Rn}\end{array}$ \\
\hline${ }^{212} \mathrm{Bi}$ & $1,01 \mathrm{~h}$ & $\begin{array}{l}\beta 2,25 \\
\alpha 6,09\end{array}$ & $\begin{array}{l}\text { (64) } \\
\text { (36) }\end{array}$ & 0,04 & TGI & 0,26 & 31 & \\
\hline${ }^{212} \mathrm{Po}$ & $0,3 \mu \mathrm{s}$ & $\alpha 8,78$ & & & & & & \\
\hline
\end{tabular}

(1) Valeur la plus restrictive dans le cas d'un adulte (âge $>17 \mathrm{ans}$ ), (2) Les effets biologiques des ga/ radioactifs ${ }^{222} \mathrm{Rn}$ et ${ }^{220} \mathrm{R} n$ sont dus à leurs descendants solides à vie courte émetteurs alpha. L'énergic alpha totale des particules érnises qui s'exprime en J m ${ }^{-3}$, est le paramètre essentiel qui détermine la dose délivréc notamment au niveau des poumons par les descendints du radon. 


\subsection{Rayonnement tellurique}

Il a pour origine les radionucléides émetteurs gamma présents dans les roches et les sols. Le ${ }^{40} \mathrm{~K}$ et les descendants émetteurs gamma des séries de ${ }^{238} \mathrm{U}$ et du ${ }^{232} \mathrm{Th}$ sont les principaux isotopes responsables de l'irradiation externe d'origine tellurique des populations (Madelmont et al., 1984).

L'existence d'un élément chimique gazeux, le radon, dans les deux familles de l'uranium et du thorium contribue à réduire, du fait de son exhalation des sols et des roches, l'exposition tellurique imputable aux émetteurs gamma postémanation présents dans les deux familles (Tab. III).

L'irradiation d'origine tellurique est en moyenne de $60 \mathrm{nGy} \mathrm{h}^{-1}$. Elle peut varier entre 10 et $200 \mathrm{nGy} \mathrm{h}^{-1}$ en fonction des concentrations en ${ }^{40} \mathrm{~K}$ et en radionucléides émetteurs gamma des familles de ${ }^{238} \mathrm{U}$ et du ${ }^{232} \mathrm{Th}$ présents dans les sols. Une autre cause de variation rapide est due au dépôt sur le sol, en cas de pluie, des descendants solides émetteurs gamma post-émanation, du ${ }^{222} \mathrm{Rn}$ notamment.

La dose moyenne annuelle associée à l'irradiation naturelle d'origine tellurique est estimée à $74 \mu \mathrm{Sv}$ pour un individu séjournant $20 \%$ de son temps à l'extérieur des bâtiments.

\subsection{Autres causes d'irradiation naturelle des populations}

La présence de radionucléides émetteurs gamma dans les matériaux de construction ainsi que de radon et de thoron dans l'air des bâtiments conduit à des doses moyennes annuelles mondiales respectivement de $410,700^{14}$ et $150 \mu \mathrm{Sv}$, pour un individu qui séjourne en moyenne $80 \%$ de son temps à l'intérieur des bâtiments.

Les niveaux de radioactivité naturelle des matériaux de construction et les concentrations en radon et thoron dans les bâtiments ne sont pas abordés dans cet article. Pour la France, des informations sont disponibles dans les publications (Métivier et Robé, 1998 ; Robé et al., 1992a), une étude des zones potentiellement exposées à de fortes concentrations en radon étant en cours ${ }^{15}$.

\footnotetext{
${ }^{14}$ Pour une concentration moyenne en ${ }^{222} \mathrm{Rn}$ dans l'air de $40 \mathrm{~Bq} \mathrm{~m}^{-3}$ et un facteur d'équilibre de 0,4 entre le ${ }^{222} \mathrm{Rn}$ et ses descendants à vie courte.

${ }^{15}$ Collaboration BRGM/IPSN dans le cadre du programme Environnement et Santé
} 


\section{Méthodes de sélection et de collecte des données}

Dresser un inventaire en France des niveaux de radioactivité naturelle dans l'environnement qui ne soient pas perturbés par l'action de l'homme à l'extérieur des habitations, implique d'identifier les installations susceptibles de modifier le bruit de fond radioactif d'origine naturelle.

À cet effet, les organismes qui ont participé à cet inventaire ont reçu un jeu de tableaux (Fig. 1) où les résultats radioactifs relatifs à chaque constituant ou composante de la biosphère devaient être reportés en fonction de leur zone d'origine (Tab. IV).

- types de mesures réalisées par les organismes,

- caractéristiques des radionucléides naturels,

- questionnaire adresse aux organismes,

- mesures disponibles sélectionnés

\section{- Méthode de présentation}

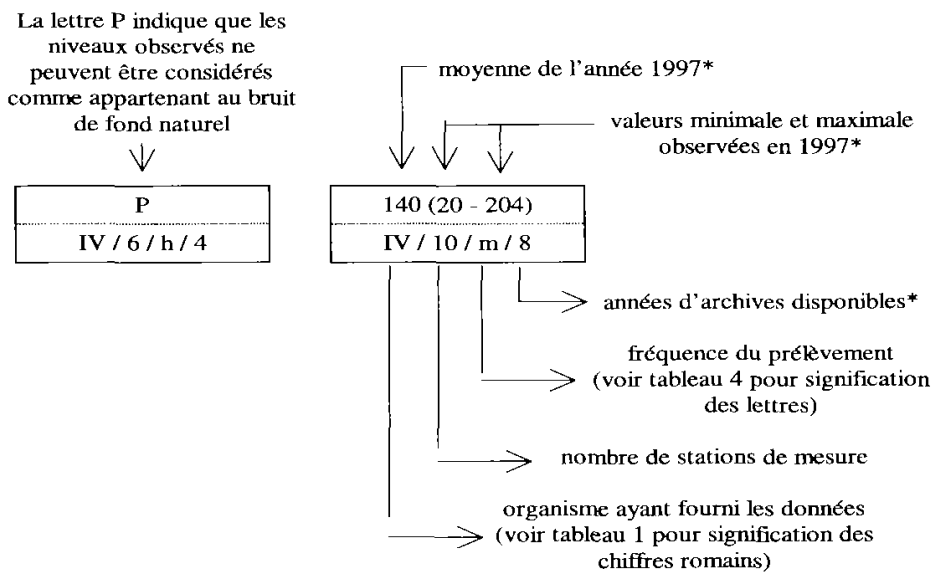

* Pour les mesures occasionnelles, c'est le nombre d'années pendant leur réalisation qui est pris en compte.

Figure 1 - Modèle de présentation des données regroupées dans les tableaux VI à XVI.

Presentation of the data grouped in Tables VI to XVI. 


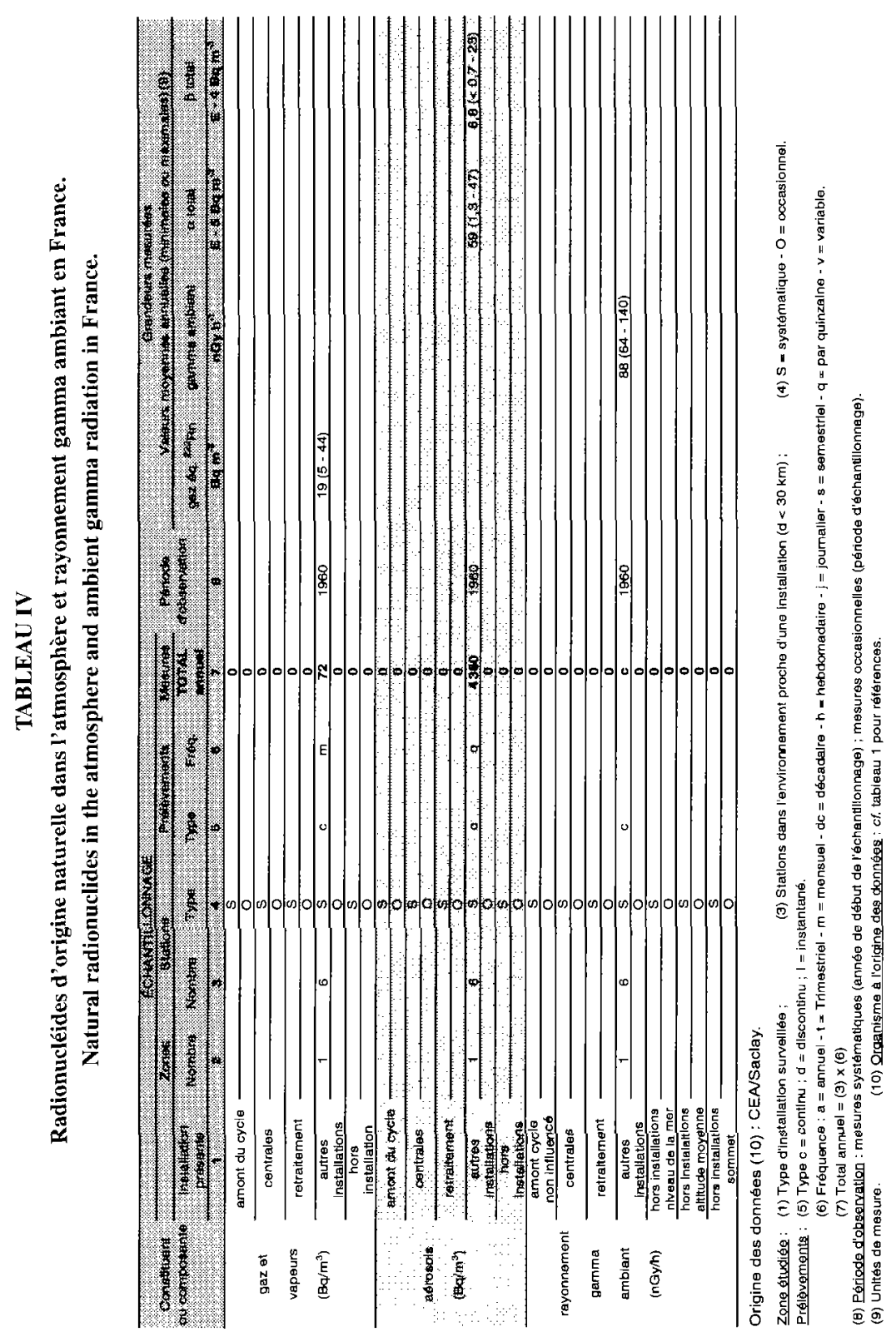


Cinq zones étaient distinguées :

- celles dotées d'installations nucléaires liées à l'amont du cycle du combustible: mines, usines de traitement du minerai, de conversion et d'enrichissement de l'uranium, et de fabrication des combustibles dont les activités peuvent modifier la distribution des isotopes de la famille de l'uranium et du thorium dans l'environnement ${ }^{16}$;

- celles dotées de centrales électronucléaires qui rejettent, outre des radionucléides artificiels, du tritium et du carbone-14 ;

- celles où sont présentes des usines de retraitement de combustibles irradiés ${ }^{17}$, qui rejettent aussi du tritium et du carbone-14 mais avec des activités beaucoup plus importantes que celles mises en jeu sur un site EDF équipé de réacteurs à eau pressurisée (REP) ;

- celles où sont implantés les centres du Commissariat à l'énergie atomique (CEA), les bases de la Marine nationale ou les centres de stockage de déchets de l'Agence nationale pour la gestion des déchets radioactifs (ANDRA). Selon la mission de ces établissements, des isotopes radioactifs naturels tant cosmogéniques que telluriques peuvent y être détenus, produits et/ou rejetés ;

- celles exemptes de toute INB pour lesquelles il convient toutefois de s'assurer qu'une installation industrielle non nucléaire ne manipule pas ou ne détient pas des matériaux radioactifs susceptibles de perturber les niveaux de radioactivité naturelle dans l'environnement.

Les figures 2 et 3 présentent respectivement la localisation des principaux sites où sont effectuées des mesures systématiques, soit in situ du rayonnement gamma ambiant, soit différées en laboratoire de la radioactivité d'échantillons prélevés dans l'environnement.

Compte tenu de l'abondance des données, de leur variation faible d'une année sur l'autre pour un radioélément donné et un constituant donné, de méthodes d'échantillonnage et de mesure différentes mises en auvre par les organismes contactés, le recueil des données s'est effectué en tenant compte des prescriptions suivantes :

- pour les mesures à caractère systématique, liées en général au contrôle réglementaire des installations nucléaires et à la surveillance de l'environnement, seules les observations faites en 1997 avaient été prises en compte. Pour les mesures à caractère occasionnel associées plutôt aux recherches et expertises, la période de référence prise en compte correspondait à celle d'exécution de ces travaux,

\footnotetext{
${ }^{16}$ Les usines de production d'cngrais phosphatés ou qui manipulent des matériaux contenant du thorium ont été rallachées à cette catégorie d'installations.

${ }^{17}$ Il est à noter que le retraitement des combustibles nucléaires a élć arrête à Marcoule fin 1998
} 


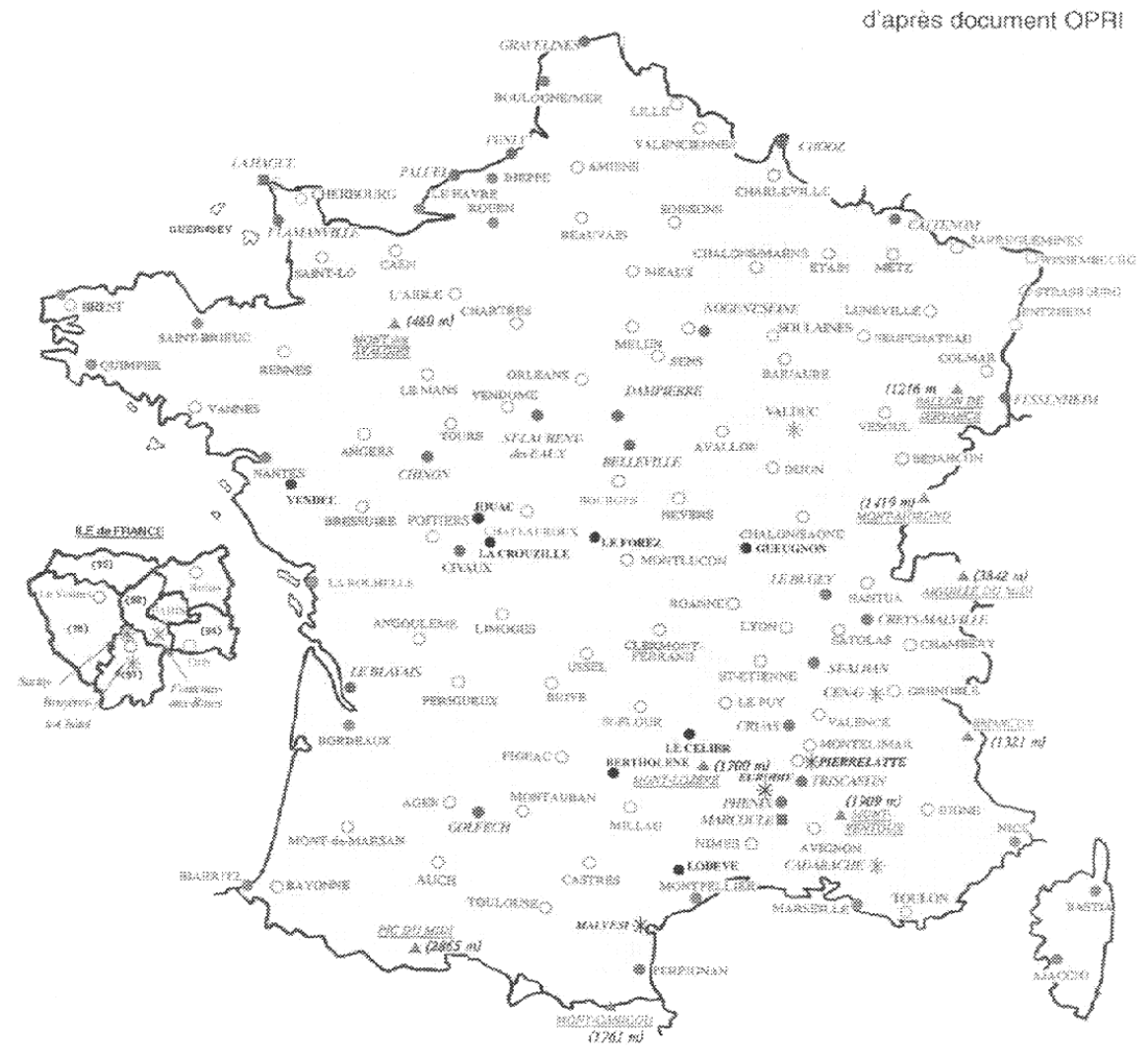

Légenda ?

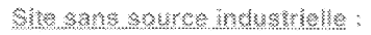

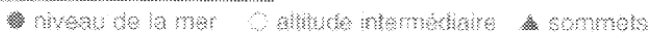

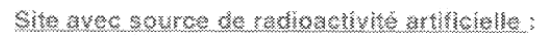

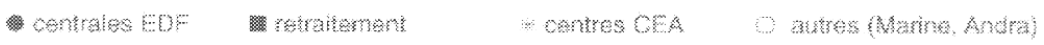

Site avec source de radioactivite naturella :
sites miniers
comersion
alitres

Figure 2 - Principaux sites de mesure permanente in situ du rayonnement gamma ambiant par balise(s) et/ou dosimètres.

Main permanent measuring sites for in situ measurement of ambient gamma radiation by probe or dosimetre. 


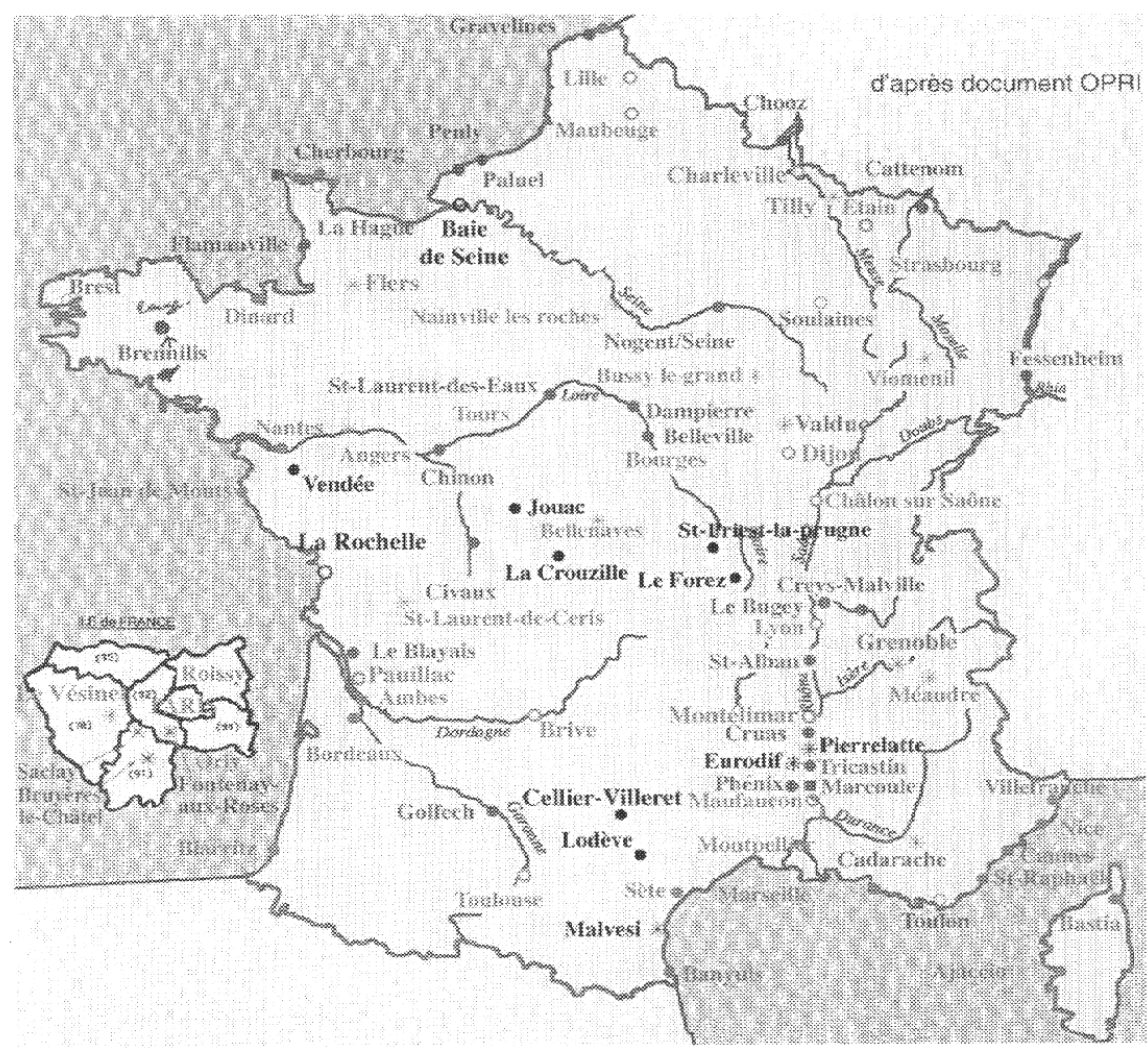

\section{Logende :}

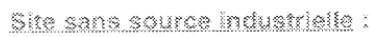

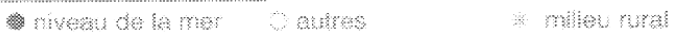

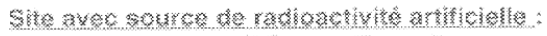

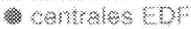

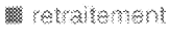
* cantus ces

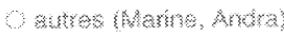

Síte avec source de radioactivité naturelle:

* witas miniers * conversion

enticinssemert

o autes inhosphates, ceramioues?

Figure 3-Principaux sites de mesures systématiques de la radioactivité d'échantillons de l'environnement. Mesures différées en laboratoire.

Main permanent measuring sites for radioactivity in environmental samples. Measurement postponed to the laboratory. 
- dans les deux cas précités, à partir de l'ensemble des valeurs disponibles était calculée la moyenne et précisées les valeurs minimale et maximale observées en 1997 (mesures systématiques) ou sur la période particulière consacrée à l'étude ou à l'expertise (mesures occasionnelles),

- enfin, seules les données correspondant à des mesures sélectives qui permettaient l'identification des isotopes naturels ont été recensées. D'autre part, seules les mesures dont le résultat n'était pas perturbé par une activité humaine ont été retenues.

\section{Résultats}

\subsection{Remarques générales}

Les plans d'échantillonnage et de mesure ont généralement pour objectif la détection des radioéléments d'origine artificielle. Seules la surveillance et l'étude des sites liés à la manipulation de matériaux contenant de l'uranium et du thorium se traduisent par la mise en œuvre d'une méthodologie systématiquement orientée vers la détection des radioéléments d'origine naturelle.

Les isotopes naturels sont souvent des radionucléides émetteurs alpha, bêta ou gamma de faible énergie (Tabs. II et III) dont la détection nécessite le recours à des techniques radiochimiques associées à des mesures nucléaires des rayonnements émis où les causes de perturbation du résultat de la mesure sont nombreuses ${ }^{18}$ (Robé et al., 1992b).

En conséquence, la mesure sélective d'isotopes naturels est loin d'être systématique sur les échantillonnages réalisés. D’autre part, la priorité accordée à la recherche des radioéléments artificiels aboutit souvent à l'absence d'archivage exploitable des données sur les radioéléments naturels et ce même lorsque les mesures ont été effectuées.

Les résultats présentés dans ce document ne constituent en conséquence qu'une fraction des données existantes relatives à la radioactivité naturelle dans l'environnement en France. Selon la zone et le constituant concernés, les résultats retenus dans ce document émanent de l'organisme dont les archives apparaissaient les plus complètes ${ }^{19}$.

\footnotetext{
${ }^{18}$ Par exemple, lc phénomène d'auto-atténuation variable du rayonnement émis en fonction des caractéristiques nucléaires du radionucléide recherché et de la nature de l'échantillon mesuré.

${ }^{19}$ Nombre d'observations disponibles, sensibilité des mesures et djversité des isotopes identifiés.
} 


\section{TABLEAU V}

Rayonnement gamma ambiant en France $\left(n G y ~^{-1}\right)$. Niveaux non perturbés par l'action de l'homme. Mesures par balises ou dosimètres renouvelés périodiquement.

Ambient gamma radiation in France $\left(n G y h^{-1}\right)$. Levels free from human interference. Measurements by probes or dosimeters that are regularly renewed.

\begin{tabular}{|c|c|c|c|c|}
\hline (2.) & (20) & Whonter & Horabre do whor & 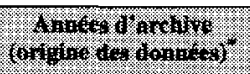 \\
\hline \multicolumn{2}{|l|}{ Amont du cycle } & $\begin{array}{c}140 \\
(80-240)\end{array}$ & 13 & $9($ II $)$ \\
\hline \multicolumn{2}{|c|}{ 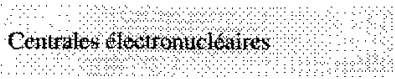 } & $800-160)$ & 21 & $9 \mathrm{x})$ \\
\hline \multirow[b]{2}{*}{ Retraitement } & Marcoule & $\begin{array}{c}63 \\
(38-91)\end{array}$ & 6 & 42 (VII) \\
\hline & La Hague & $\begin{array}{c}90 \\
(30-170)\end{array}$ & 13 & 33 (VII) \\
\hline \multirow{3}{*}{$\begin{array}{l}\text { Autres } \\
\text { installattons }\end{array}$} & Eentre of stelay & $68+40$ & 6 & 390 \\
\hline & Certrede $14 \mathrm{M}_{\text {and }}$ he & $\begin{array}{l}290 \\
(69-140)\end{array}$ & 21 & 26010 \\
\hline & $\begin{array}{l}\text { Niveau de } \\
\text { la mer }\end{array}$ & $\begin{array}{c}80 \\
(50-150)\end{array}$ & 20 & $9(X \vee I)$ \\
\hline \multirow[t]{2}{*}{$\begin{array}{c}\text { Hors } \\
\text { installations }\end{array}$} & $\begin{array}{l}\text { Altitude } \\
\text { moyenne }\end{array}$ & $\begin{array}{c}90 \\
(40-180)\end{array}$ & 53 & $9(\mathrm{XVI})$ \\
\hline & Sommets & $\begin{array}{c}140 \\
(70-300)\end{array}$ & 9 & $9(X V I)$ \\
\hline
\end{tabular}

* $C f$. figure I et tableau $\mathbf{I}$.

\subsection{Irradiation totale gamma ambiante}

Les résultats proviennent de nombreuses stations réparties sur l'ensemble du territoire (Fig. 2). Les mesures sont réalisées soit, in situ à l'aide de balises qui délivrent une information permanente, soit par des dosimètres thermoluminescents renouvelés périodiquement.

À titre indicatif, le réseau TELERAY comporte 152 stations équipées de balises. Par ailleurs, chacun des sites nucléaires français importants dispose de 5 à 40 stations de mesure dotées de balises et/ou de dosimètres (OPRI, 1997).

Les niveaux d'irradiation présentés (Tab. V) correspondent en chaque station à la somme des irradiations dues aux rayonnements cosmique et tellurique.

Les valeurs d'irradiation les plus élevées sont logiquement observées dans les stations implantées sur des sommets et dans les régions granitiques telles que le nord-Limousin où, dans les deux situations, la valeur moyenne observée est de 
$140 \mathrm{nGy} \mathrm{h}^{-1}$. Ces résultats traduisent l'influence de l'altitude sur l'accroissement de la composante d'origine cosmique dans le cas des mesures faites sur les sommets (Fig. 1), et celle des roches granitiques sur l'accroissement de la composante d'origine tellurique dans le cas du Limousin.

En règle générale, c'est la composante tellurique qui contribue de façon prépondérante à l'irradiation gamma totale ambiante comme le montrent les résultats issus des 20 stations en bord de mer gérées par l'OPRI : compte tenu d'une irradiation cosmique moyenne de $31 \mathrm{nGy} \mathrm{h}^{-1}$ à cette altitude, la composante tellurique peut être estimée à $49 \mathrm{nGy} \mathrm{h}^{-1}$ avec des valeurs susceptibles de fluctuer entre 19 et $119 \mathrm{nGy} \mathrm{h}^{-1}$.

Lorsque la radioactivité naturelle des sols est connue, il est possible d'évaluer le débit de dose de la composante tellurique ${ }^{20}$ et par soustraction de cette valeur à l'irradiation totale mesurée ${ }^{21}$, d'en déduire la contribution due au seul rayonnement cosmique. À titre d'exemple, pour le site de Saclay, ce calcul conduit à une irradiation d'origine tellurique estimée à $54 \mathrm{nGy} \mathrm{h}^{-1}$ pour une irradiation totale moyenne de $88 \mathrm{nGy}^{-1}$ d'où une contribution de la composante cosmique de $34 \mathrm{nGy} \mathrm{h}^{-1}$. Il en résulte des doses annuelles sensiblement équivalentes associées à ces deux sources naturelles pour un individu séjournant $20 \%$ de son temps à l'extérieur des bâtiments :

- irradiation tellurique : $54 \mathrm{nGy} \mathrm{h}^{-1} \times 8760 \mathrm{~h} \times 0,2 \times 0,7 \mathrm{~Sv} \mathrm{~Gy}^{-1^{22}}=66 \mu \mathrm{Sv}$, - $\quad$ irradiation cosmique : $34 \mathrm{nGy} \mathrm{h}^{-1} \times 8760 \mathrm{~h} \times 0,2 \times 1 \mathrm{~Sv} \mathrm{~Gy}^{-1^{23}}=60 \mu \mathrm{Sv}$.

Les valeurs moyennes françaises sont comparables aux valeurs mondiales. Ainsi, à partir des analyses effectuées sur les sols récoltés dans l'environnement immédiat des 21 sites électronucléaires français (Tab. VI), l'irradiation tellurique naturelle peut être estimée à $58 \mathrm{nGy} \mathrm{h}^{-1}$, valeur identique à la moyenne mondiale pondérée proposée par l'UNSCEAR (1988, 1993).

Dans l'évaluation des irradiations externes et des doses annuelles qui y sont associées, chaque site doit être considéré en tenant compte de ses particularités. Ainsi, l'irradiation tellurique naturelle calculée sur des plages proches de l'usine de La Hague n'atteint que $24 \mathrm{nGy} \mathrm{h}^{-1}$, compte tenu de la radioactivité naturelle

\footnotetext{
${ }^{20}$ Des coelficients de $0,0417,0,463$ et $0,604 \mathrm{nGy} \mathrm{h}{ }^{-1} / \mathrm{Bq} \mathrm{kg}^{-1}$ sont proposés par Saito (Saito et Jacob, 1995 ) pour évaluer le rayonnement tellurique à parlir des concentrations respectives en ${ }^{4(9} \mathrm{K},{ }^{238} \mathrm{U}$ et ${ }^{232} \mathrm{Th}$ présentes dans les sols.

${ }^{21}$ En France, les radioćléments artificiels émetteurs gamma présents dans les sols ct les sédiments ont en général une activité marginale par rapport à celles imputables aux radioéléments naturels.

${ }^{22}$ Valeur moyenne du coefficient de conversion de dose retenu pour des adultes pour le rayonnement d'origine tellurique (UNSCEAR, 1988, 1993).

${ }^{23}$ Cocfficient de conversion pris égal à l'unité compte tenu de la dominante des muons dans le rayonncment cosmique au niveau du sol (UNSCEAR, 1988, 1993).
} 


\section{TABLEAU VI}

Radioactivité naturelle des sols en France $\left(\mathrm{Bq} \mathrm{kg}^{-1}\right.$ sec)* niveaux non perturbés par l'action de l'homme (notations : $c f$. Fig. 1 et Tab. I).

Natural radioactivity of soil in France $\left(B q \mathrm{~kg}^{-1} \mathrm{dry} \text { weight }\right)^{*}$. Levels free from human interference (notations: $c f$. Fig. 1 and Tab. I).

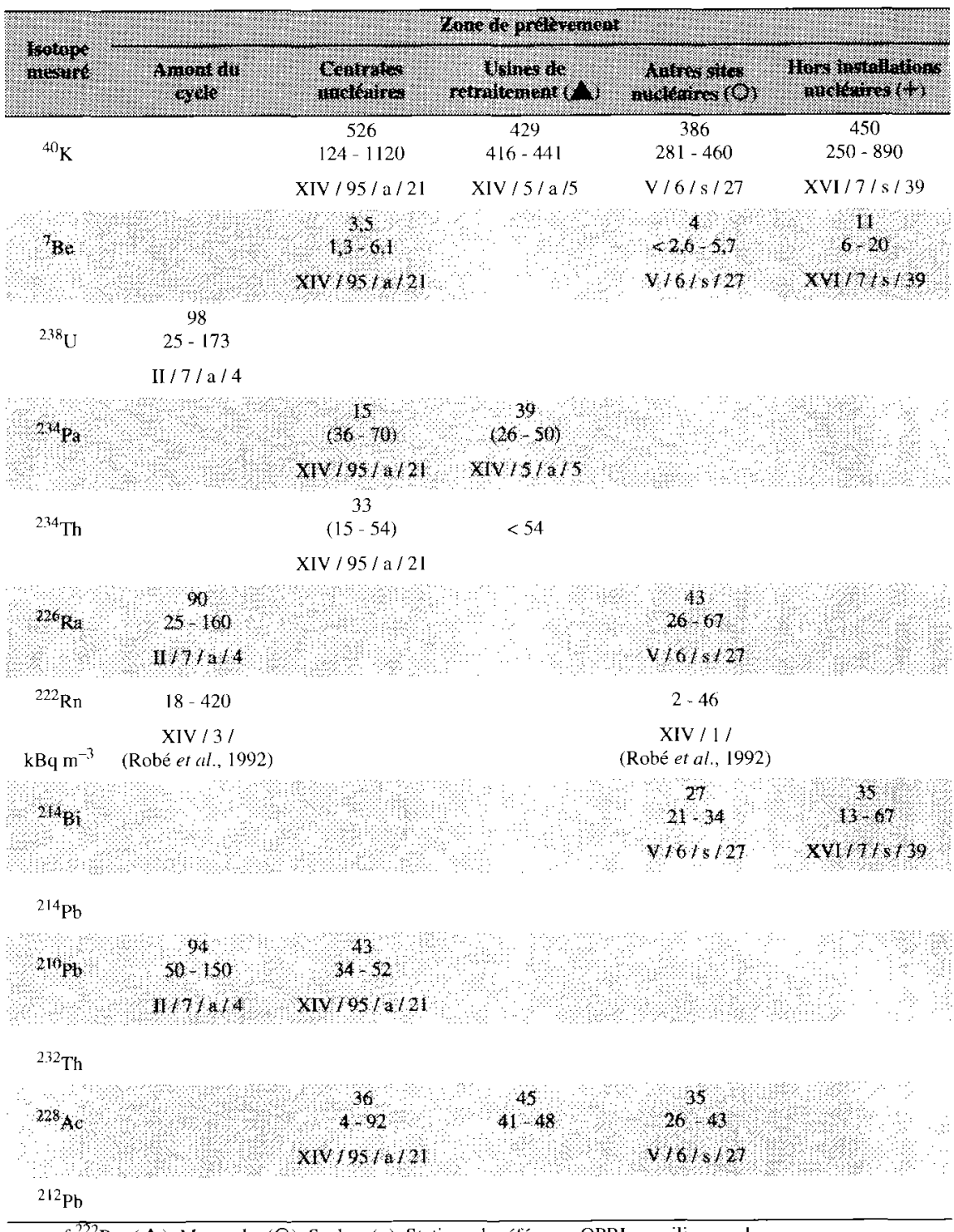

* sauf ${ }^{222} \mathrm{Rn},(\boldsymbol{\Delta})$ Marcoule, (O) Saclay, (+) Stations de référence OPRI en milieu rural. 
assez faible présente dans les sédiments marins sableux présents sur l'estran (Tab. VIII) que cette estimation soit faite à partir des résultats de mesures effectuées par la Marine nationale ou par l'ACRO. Le calcul des doses doit enfin s'appuyer sur la connaissance du mode de vie des populations locales. Ainsi un adulte qui effectuerait quotidiennement une promenade d'une heure sur ces plages recevrait une dose annuelle due à la composante tellurique naturelle de $6 \mu \mathrm{Sv}^{24}$ et de $12 \mu S v^{25}$ liée au rayonnement cosmique.

\subsection{Radioactivité naturelle des sols et des sédiments}

Les sols et les sédiments qui résultent de l'altération des roches sous l'effet du climat constituent la source de radioactivité naturelle à laquelle se trouve le plus directement exposé l'homme (Tabs. VI-VIII). Leurs teneurs en radioéléments naturels déterminent l'importance de l'irradiation externe d'origine tellurique. Mais leurs caractéristiques radioactives et pédologiques vont aussi conditionner l'importance des transferts des radionucléides depuis le sol ou les sédiments, vers l'atmosphère (exhalation du radon, remise en suspension de particules radioactives), les végétaux terrestres cultivés, les bioindicateurs mais également influencer le taux de lessivage des radionucléides par les eaux de ruissellement.

Le ${ }^{40} \mathrm{~K}$ est, quel que soit le site et le type de prélèvement, l'isotope radioactif naturel le plus abondant: sa concentration, de 350 à $600 \mathrm{~Bq} \mathrm{~kg}^{-1} \mathrm{sec}$ (Gontier, 1998), dépasse en général d'un facteur 10 ou plus celles de tous les autres isotopes naturels appartenant aux familles de ${ }^{238} \mathrm{U}$ ou du ${ }^{232}$ Th qui se situent en général entre 10 et $50 \mathrm{~Bq} \mathrm{~kg}^{-1} \mathrm{sec}$. Toutefois, dans les zones uranifères, en particulier dans les sols, les concentrations des isotopes de la famille de ${ }^{238} \mathrm{U}$ sont environ deux fois supérieures à celles habituellement observées dans les autres zones. Cette différence se traduit aussi au niveau des activités en ${ }^{222} \mathrm{Rn}$ dans les pores des sols des régions granitiques où elles peuvent varier entre 18 et $420 \mathrm{kBq} \mathrm{m}^{-3}$ ce qui correspond à des concentrations 10 fois supérieures à celles observées dans des zones sédimentaires telles que le Bassin parisien (Robé et al., 1992b).

Dans une même région géographique, les concentrations en isotopes naturels vont en outre varier en fonction de la granulométrie des matériaux prélevés. L'abondance de particules fines dans un sol ou un sédiment se traduit en général par des concentrations plus élevées comme le montrent les activités des vases récoltées dans la rade de Cherbourg par rapport aux sédiments sableux récoltés sur les plages du nord-Contentin (Schmidt et al., 1998).

\footnotetext{
${ }^{24} 365 \mathrm{j} \times 1 \mathrm{I} \mathrm{h} \mathrm{j}^{-1} \times 24 \mathrm{nGy} \mathrm{h}^{-1} \times 0,7 \mathrm{~Sv} \mathrm{~Gy}^{-1}=6 \mu \mathrm{Sv}$.

${ }^{25} 365 \mathrm{j} \times 1 \mathrm{~h} \mathrm{j}^{1} \times 32 \mathrm{nGy} \mathrm{h}^{-1} \times 1 \mathrm{~Sv} \mathrm{~Gy}{ }^{-1}=12 \mu \mathrm{Sv}$.
} 


\section{TABLEAU VII}

Radioactivité naturelle des sédiments d'eau douce en France ( $\mathrm{Bq} \mathrm{kg}^{-1}$ sec) niveaux non perturbés par l'action de l'homme (notations : $c f$. Fig. 1 et Tab. I).

Natural radioactivity of freshwater sediments in France ( $\left.\mathrm{Bq} \mathrm{kg}^{-1} \mathrm{dry}\right)$. Levels free from human interference (notations: $c f$. Fig. 1 and Tab. I).

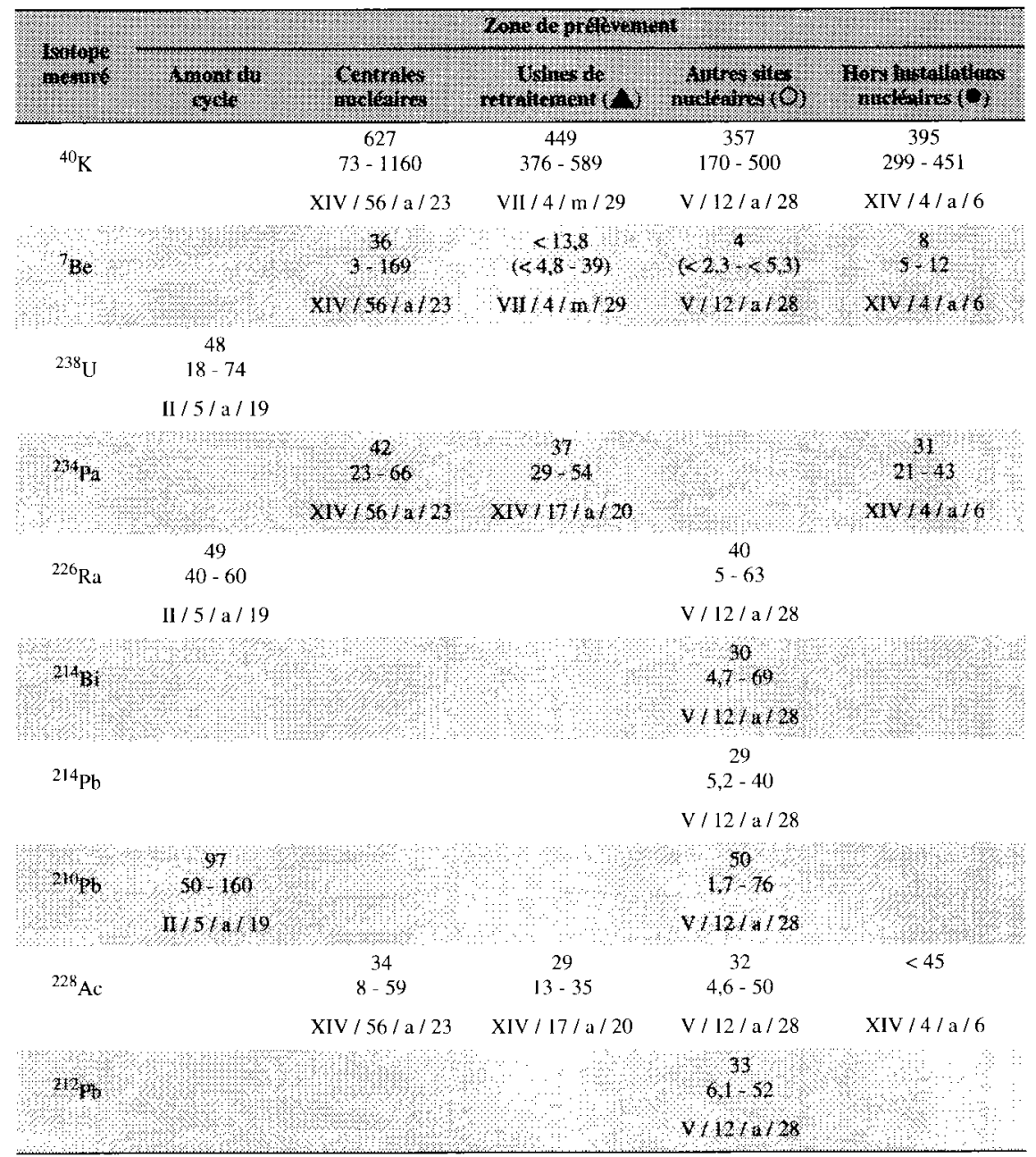

(A) Marcoule, (O) Saclay, (•) Stations observatoires IRSN. 


\section{TABLEAU VIII}

Radioactivité naturelle des sédiments marins en France $\left(\mathrm{Bq} \mathrm{kg}^{-1} \mathrm{sec}\right)$ niveaux non perturbés par l'action de l'homme (notations : $c f$. Fig. 1 et Tab. I).

Natural radioactivity of marine sediments in France $\left(\mathrm{Bq} \mathrm{kg}^{-1} \mathrm{dry}\right)$. Levels free from human interference (notations: $c f$. Fig. 1 and Tab. I).

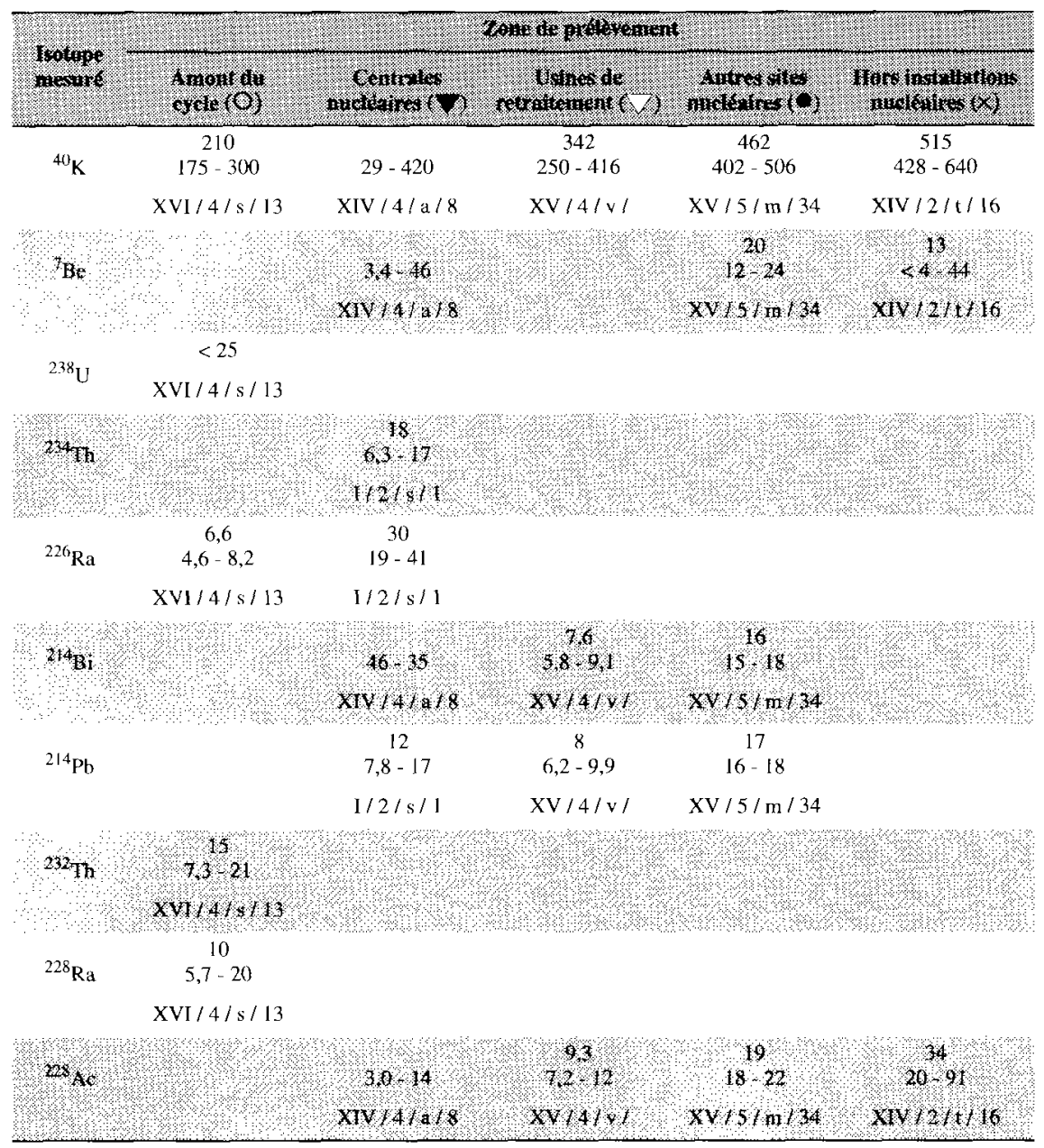

( ) La Hague - Sables sur l'estran, ( $\boldsymbol{V}$ ) Centrales bord de mer EDF, (O) Baie de Seine, ( $x$ ) Observatoire marin en Méditerranée, (•) Rade de Cherbourg. 
Enfin, le bruit de fond naturel dû aux isotopes des familles de l'uranium et du thorium peut être perturbé par diverses activités humaines. Si celles-ci augmentent de façon significative les concentrations en radioéléments naturels sur un site, la mise en évidence d'une pratique peut être aisé ${ }^{26}$. Mais en règle générale, pour les installations de l'amont du cycle du combustible où se réalisent des opérations d'extraction du minerai, de séparation ${ }^{238} \mathrm{U} /{ }^{226} \mathrm{Ra}$ et de stockage des déchets riches en ${ }^{226} \mathrm{Ra}$ et ses descendants, cette mise en évidence peut s'avérer délicate, notamment en milieu terrestre (Bernhard et al., 1995). Il est en effet indispensable d'identifier des stations de référence où l'action de l'homme n'a pas introduit de perturbation, pour pouvoir évaluer l'impact additionnel que peuvent induire ces différentes pratiques.

Les données recueillies montrent toutefois que l'effort d'échantillonnage ne porte pas préférentiellement sur les sols et les sédiments émergés dont la connaissance est pourtant prioritaire pour l'évaluation des transferts et des doses. Sur le plan analytique, les mesurages entrepris ne permettent pas toujours d'identifier systématiquement les trois radioéléments telluriques essentiels $\left({ }^{40} \mathrm{~K},{ }^{238} \mathrm{U},{ }^{232} \mathrm{Th}\right)$ ainsi que leurs descendants à vie longue $\left({ }^{226} \mathrm{Ra},{ }^{228} \mathrm{Ra},{ }^{210} \mathrm{~Pb}\right)$ ou les radionucléides émetteurs gamma responsables du rayonnement tellurique $\left({ }^{214} \mathrm{~Pb},{ }^{214} \mathrm{Bi}\right.$, ${ }^{212} \mathrm{~Pb}$ ). La connaissance des rapports des activités de ces isotopes constitue autant d'indices pour évaluer l'impact d'une pratique. Ainsi le rapport ${ }^{238} \mathrm{U} /{ }^{232} \mathrm{Th}$ varie entre 0,4 et 10 dans les roches alors que dans les rejets des mines françaises, il se caractérise par une valeur supérieure à 100 (Zettwoog et al., 1997).

\subsection{Radioactivité naturelle de l'atmosphère}

La source principale d'irradiation naturelle des populations est liée à la présence des descendants émetteurs alpha à vie courte du radon (Tab. IX) (Renoux, 1984). Par exemple, sur le site de Saclay, les doses annuelles ${ }^{27}$ liées à l'inhalation des radioéléments naturels peuvent être estimées à $120 \mu \mathrm{Sv}$ pour le ${ }^{222} \mathrm{Rn}^{28}$ et $6 \mu \mathrm{Sv}$ pour le ${ }^{210} \mathrm{~Pb}^{29}$. Elles sont très inférieures pour le ${ }^{7} \mathrm{Be}(0,2 \mathrm{nSv}), \mathrm{le}^{40} \mathrm{~K}(0,04 \mathrm{nSv})$ et le ${ }^{22} \mathrm{Na}(0,001 \mathrm{nSv})$. La mesure de l'énergie alpha potentielle (EAP) sur certains sites permet d'aboutir à une évaluation plus précise des doses respectives dues aux descendants du radon par la mesure de ces derniers. La nouvelle directive européenne (JOCE, 1996) fait explicitement référence aux mesures d'EAP pour

\footnotetext{
${ }^{26}$ Les mexures réalisées par l'ACRO à St-Nicolas d'Aliermont par exemple (ancienne usine manipulant du radium) donnent des teneurs maximales en ${ }^{226} \mathrm{Ra}$ dans les sols de l'ordre de $150000 \mathrm{~Bq} \mathrm{~kg}{ }^{-1}$.

${ }^{27}$ Les doses efficaces calculées dans ce paragraphe correspondent au cas d'un adulte en activité (débit respiratoire $1,2 \mathrm{~m}^{3} \mathrm{~h}^{\mathrm{l}}$ ).

${ }^{28}$ Pour le ${ }^{222} \mathrm{Rn}$ facteur de conversion de dose retenu : $6 \mathrm{nSv} / \mathrm{Bq} \mathrm{h} \mathrm{m}^{-3}$ avec un coefficient d'équilibre de 0,6 avec ses descendints (JOCE, 1996) $19 \mathrm{~Bq} \mathrm{~m} \mathrm{~m}^{-3} \times 8760 \mathrm{~h} \times 0,2 \times 0,6 \times 6 \mathrm{nSv} / \mathrm{Bq} \mathrm{h} \mathrm{m}{ }^{-3}$.

${ }^{29} 498 \times 10^{-6} \mathrm{~Bq} \mathrm{~m} \mathrm{~m}^{-3} \times 8760 \mathrm{~h} \times 0,2 \times 1,2 \mathrm{~m}^{3} \mathrm{~h}^{-1} \times 5,6 \times 10^{-6} \mathrm{~Sv} / \mathrm{Bq}$ (Tab. III).
} 


\section{TABLEAU IX}

Radioactivité naturelle de l'atmosphère en France, niveaux non perturbés par l'action de l'homme (notations : $c f$. Fig. 1 et Tab. I).

Natural radioactivity of atmosphere in France. Levels free from human interference (notations: cf. Fig. 1 and Tab. I).

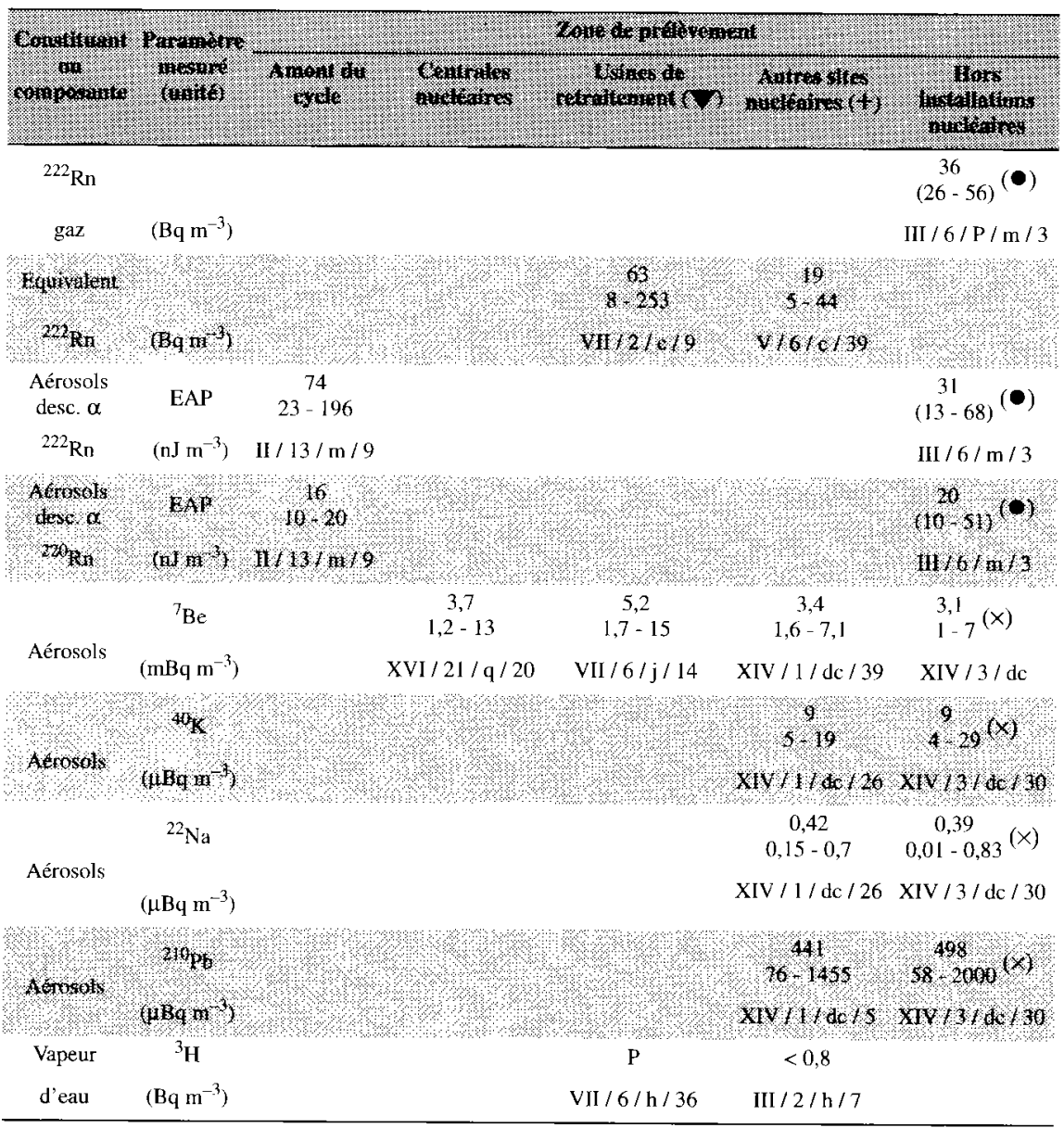

(•) Site dans l'est de la France du futur laboratoire souterrain de l'ANDRA (mesure d'1/2 heure de jour et de nuit). (+) Site de Saclay. ( $x$ ) Station observatoire atmosphérique de l'IRSN (Flers - Charleville - Dijon). ( $\nabla$ ) Marcoule. EAP : Energie alpha potentielle. 
l'estimation des doses liées aux descendants des ${ }^{222} \mathrm{Rn}$ et ${ }^{220} \mathrm{Rn}$. Dans le cas du site Est de la France destiné à accueillir le futur laboratoire souterrain de l'ANDRA (1998), un calcul effectué à partir de la mesure du ${ }^{222} \mathrm{Rn}$ conduit à une dose de $230 \mu \mathrm{Sv}$, alors qu'en se basant sur les mesures d'EAP, les contributions respectives des descendants du ${ }^{222} \mathrm{Rn}$ et ${ }^{220} \mathrm{Rn}$ peuvent être estimées respectivement à $60^{30}$ et $18 \mu \mathrm{Sv}^{31}$.

En dépit de la prédominance des doses par inhalation dues aux descendants du radon, la mesure de ces derniers est loin d'être systématique, sauf dans le cas des sites miniers où sa connaissance au niveau du bruit de fond naturel et des zones soumises à l'impact des travaux miniers est indispensable.

On notera également l'absence de données sur les activités volumiques de l'air en ${ }^{232}$ Th dont le coefficient de dose par inhalation est pourtant parmi les plus pénalisants (Tab. III).

\subsection{Radioactivité naturelle des aliments}

\subsubsection{Boissons}

L'activité en tritium dans les eaux, liée à sa seule production naturelle correspond à des concentrations qui se situent entre 0,12 à $0,24 \mathrm{~Bq}^{-1}$ (AIEA, 1992). Or, la plupart des concentrations mesurées dépassent cette valeur même parfois pour des stations éloignées d'installations nucléaires (Foulquier et Pally, 1982). La mobilité du tritium dans l'environnement, la contribution résiduelle des retombées dues aux explosions d'engins nucléaires dans l'atmosphère mais surtout la multiplicité des rejets liquides et gazeux de cet isotope de l'hydrogène qui suit les mouvements de l'eau, explique sa présence dans des eaux potables provenant de ressources en liaison directe ou non avec l'atmosphère (Rémy et Pellerin, 1968 ; Rémy et Lemaître, 1990). Des valeurs de plusieurs dizaines de $\mathrm{Bq}^{-1}$ sont parfois observées $^{32}$. Les mesures réalisées sur les eaux brutes superficielles ou de nappe (Tab. X) constituent un indicateur du niveau probable de pollution de l'eau potable pour cet isotope vis-à-vis duquel les traitements d'eau sont sans effet $^{33}$.

En ce qui concerne le ${ }^{40} \mathrm{~K}$ et ${ }^{238} \mathrm{U}$, les concentrations mesurées dans des eaux potables prélevées dans des zones dépourvues d'installations nucléaires conduisent à des doses par ingestion de respectivement de 0,5 et $0,3 \mu \mathrm{Sv}$.

\footnotetext{
${ }^{30} 31 \mathrm{~nJ} \mathrm{~m}{ }^{-3} \times 8760 \mathrm{~h} \times 0,2 \times 1,1 \mathrm{~Sv} / \mathrm{Bq} \mathrm{h} \mathrm{m} \mathrm{m}^{-3}=60 \mu \mathrm{Sv}$.

${ }^{31} 20 \mathrm{~nJ} \mathrm{~m}^{-3} \times 8760 \mathrm{~h} \times 0,2 \times 0,5 \mathrm{~Sv} / \mathrm{Bq} \mathrm{h} \mathrm{m}{ }^{-3}=18 \mu \mathrm{Sv}$, cf. directive 96/29 (Jammel, 1984).

${ }^{32}$ Les mesures réalisées par exemple en Seine en aval de la centráte de Nogent indiquent des concentrations en ${ }^{3} \mathrm{H}$ qui peuvent atteindrc $60 \mathrm{~Bq} 1^{-1}$ en période estivalc. En prenant en compte cette valeur et unc consommation d'eau de 2 Jitres par jour, on aboutit à une dose annuellc maximale de $365 \mathrm{j} \times 21 / \mathrm{j} \times 60 \mathrm{~Bq} / 1 \times 0,018 \times 10^{-9} \mathrm{~Sv} / \mathrm{Bq} \simeq 0,8 \mu \mathrm{S} v$.

${ }^{33}$ Le tritium, isotope de l'hydrogène, est un des constituants de la molécule d'eau $\left(\mathrm{H}_{2} \mathrm{O}\right)$, contrairement aux autres isotopes présents sous forme dissoute ou associée aux suspensions dans les eaux brutes.
} 
TABLEAU X

Radioactivité naturelle de diverses boissons en France $\left(\mathrm{Bq}^{-1}\right)$, niveaux non perturbés par l'action de l'homme (notations : $c f$. Fig. 1 et Tab. I).

Natural radioactivity of various beverages in France $\left(\mathrm{Bq}^{-1}\right)$. Levels free from human interference (notations: cf. Fig. 1 and Tab. I).

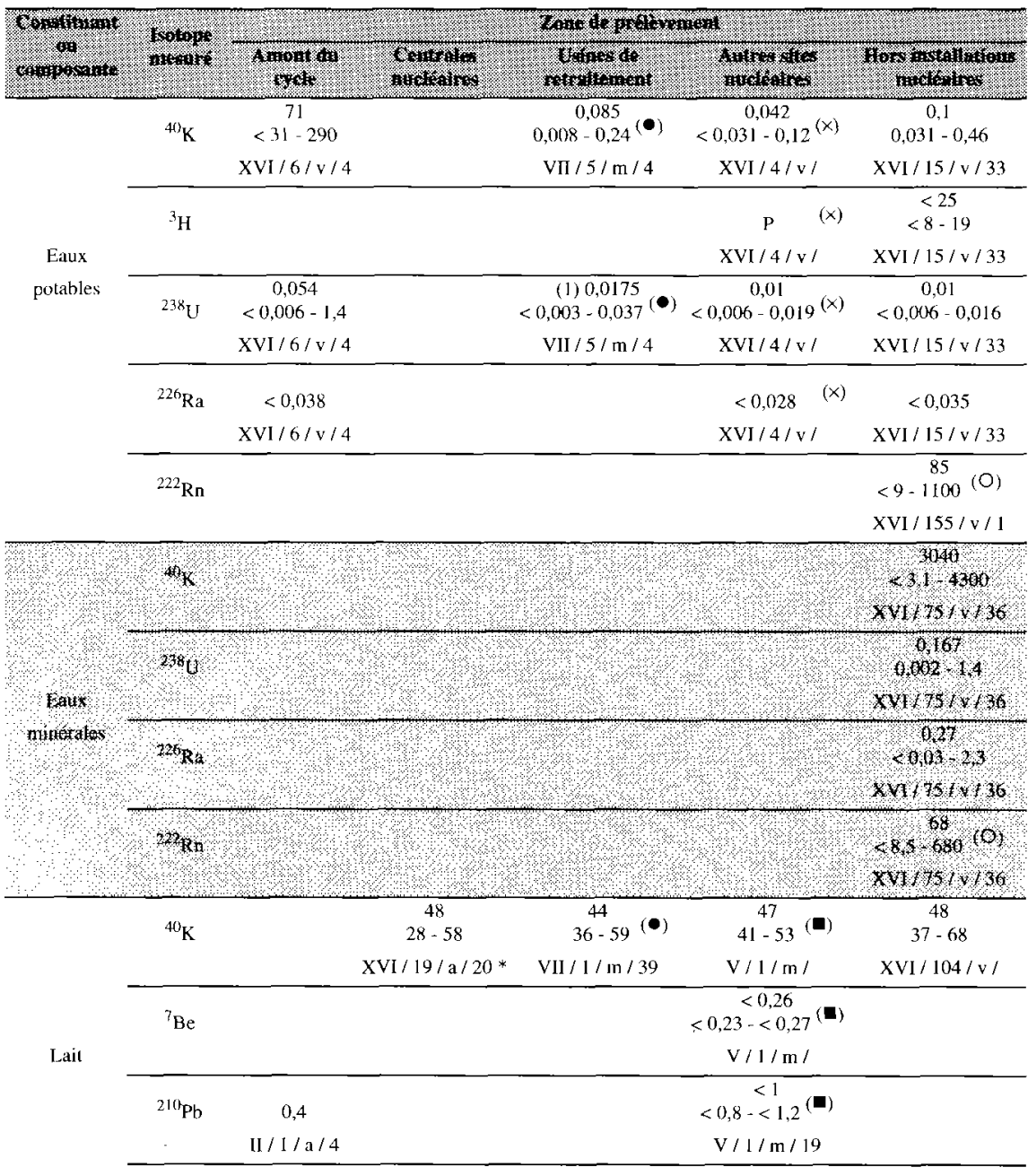

${ }^{3} \mathrm{H} \quad$ P $\quad(+)$

$\mathrm{XVI} / 5 / \mathrm{V} /$

,

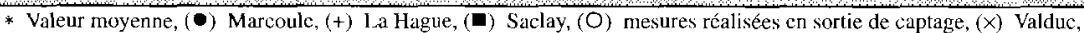

(1) dosage en uranium total $(238+235+234 \mathrm{U})$. 
Dans le cas de l'amont du cycle au niveau de ${ }^{238} \mathrm{U}$, la consommation d'eau au niveau des stations de référence conduit à une dose annuelle de $2 \mu \mathrm{Sv}$, celle associée aux ${ }^{226} \mathrm{Ra}$ pouvant atteindre $8 \mu \mathrm{Sv}$.

En ce qui concerne le lait, sur la base des données disponibles et d'une consommation moyenne annuelle en lait frais de 64 litres (Guerere et al., 1989), la dose annuelle due au ${ }^{40} \mathrm{~K}$ est de $19 \mu \mathrm{Sv}$. Le seul résultat disponible en ${ }^{210} \mathrm{~Pb}$ permet d'estimer sa contribution à $18 \mu \mathrm{Sv}$ dans le contexte d'une station de référence d'un site minier. Enfin, pour le tritium, quand les mesures sont disponibles comme dans l'environnement du site de retraitement des combustibles usés de La Hague, l'impact de l'installation est évident, la contribution à la dose annuelle atteignant moins d'un $\mu \mathrm{Sv}^{34}$.

\subsubsection{Fruits et légumes}

Les données disponibles pour les légumes feuilles permettent de comparer l'impact lié à la consommation annuelle de ces denrées ${ }^{35}$ pour différents isotopes naturels (Tabs. XI et XII). Les doses annuelles associées aux activités présentes dans ces denrées dans l'environnement des centrales françaises sont les suivantes :

${ }^{40} \mathrm{~K}=35 \mu \mathrm{Sv}, \quad{ }^{210} \mathrm{~Pb}=25 \mu \mathrm{Sv}, \quad{ }^{238} \mathrm{U}<2 \mu \mathrm{Sv}$, ${ }^{222} \mathrm{Th}=0,7 \mu \mathrm{Sv}$ et ${ }^{7} \mathrm{Be}=0,007 \mu \mathrm{Sv}$.

Les résultats obtenus au niveau des stations de référence des sites miniers sont comparables aux données recueillies dans les autres zones, les activités de ${ }^{226} \mathrm{Ra}$ disponibles permettant d'évaluer sa contribution dosimétrique à $6 \mu \mathrm{Sv}$.

Les données sur les autres catégories de légumes ou fruits dont les consommations annuelles sont au moins aussi importantes ${ }^{36}$ apparaissent moins bien renseignées.

Le nombre de résultats de mesure pour les isotopes naturels les plus importants sur le plan dosimétrique est souvent limité, sauf dans le cas des zones minières où les dosages effectués sur les stations de référence permettent d'évaluer les doses annuelles associées :

$$
{ }^{210} \mathrm{~Pb}=51 \mu \mathrm{Sv}, \quad{ }^{226} \mathrm{Ra}=6 \mu \mathrm{Sv}, \quad{ }^{238} \mathrm{U}=1 \mu \mathrm{Sv} .
$$

Pour d'autres productions telles que les céréales ${ }^{37}$ les données disponibles se limitent au seul ${ }^{40} \mathrm{~K}$.

\footnotetext{
$34730 \mathrm{l} / \mathrm{an} \times 45 \mathrm{~Bq} / 1 \times 0,018 \times 10^{-9} \mathrm{~Sv} / \mathrm{Bq} \simeq 0,6 \mu$ Sv/an.

$3546 \mathrm{~kg}$ par an (Guerere et al, 1989).

${ }^{36} 64 \mathrm{~kg} \mathrm{an}^{-1}$ pour les légumes racines et $74 \mathrm{~kg} \mathrm{an}^{-1}$ pour Jes fruits (Guerere et al., 1989)

$3747 \mathrm{~kg} \mathrm{an}^{-1}$ pour le pain par exemple.
} 


\section{TABLEAU XI}

Radioactivité naturelle des légumes en France $\left(\mathrm{Bq} \mathrm{kg}{ }^{-1}\right.$ frais), niveaux non perturbés par l'action de l'homme (notations : $c f$. Fig. 1 et Tab. I) .

Natural radioactivity of vegetables in France $\left(\mathrm{Bq} \mathrm{kg}^{-1}\right.$ fresh weight). Levels free from human interference (notations: $c f$. Fig. 1 and Tab. I) .

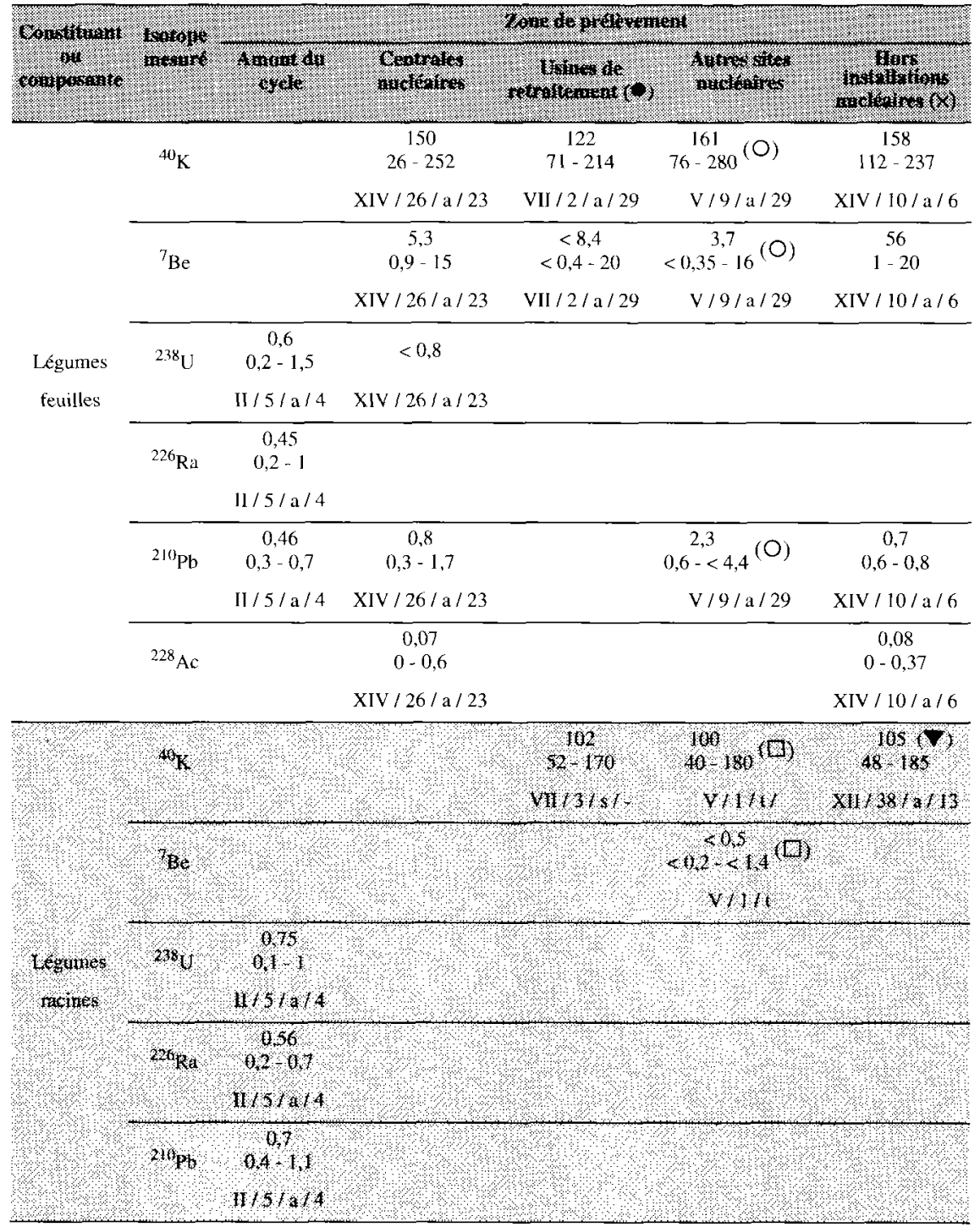

Marcoule, ( $\times$ ) Ecrins Mercantour, (O) Saclay,

Moitié nord et centre de la France, ( $\square$ ) Cadarache. 


\section{TABLEAU XII}

Radioactivité naturelle des fruits et de divers aliments en France $\left(\mathrm{Bq} \mathrm{kg}^{-1}\right.$ frais), niveaux non perturbés par l'action de l'homme (notations : $c f$. Fig. 1 et Tab. I).

Natural radioactivity of fruit and various foodstuffs in France $\left(\mathrm{Bq} \mathrm{kg}^{-1}\right.$ fresh weight). Levels free from human interference (notations: $c f$. Fig. 1 and Tab. I).

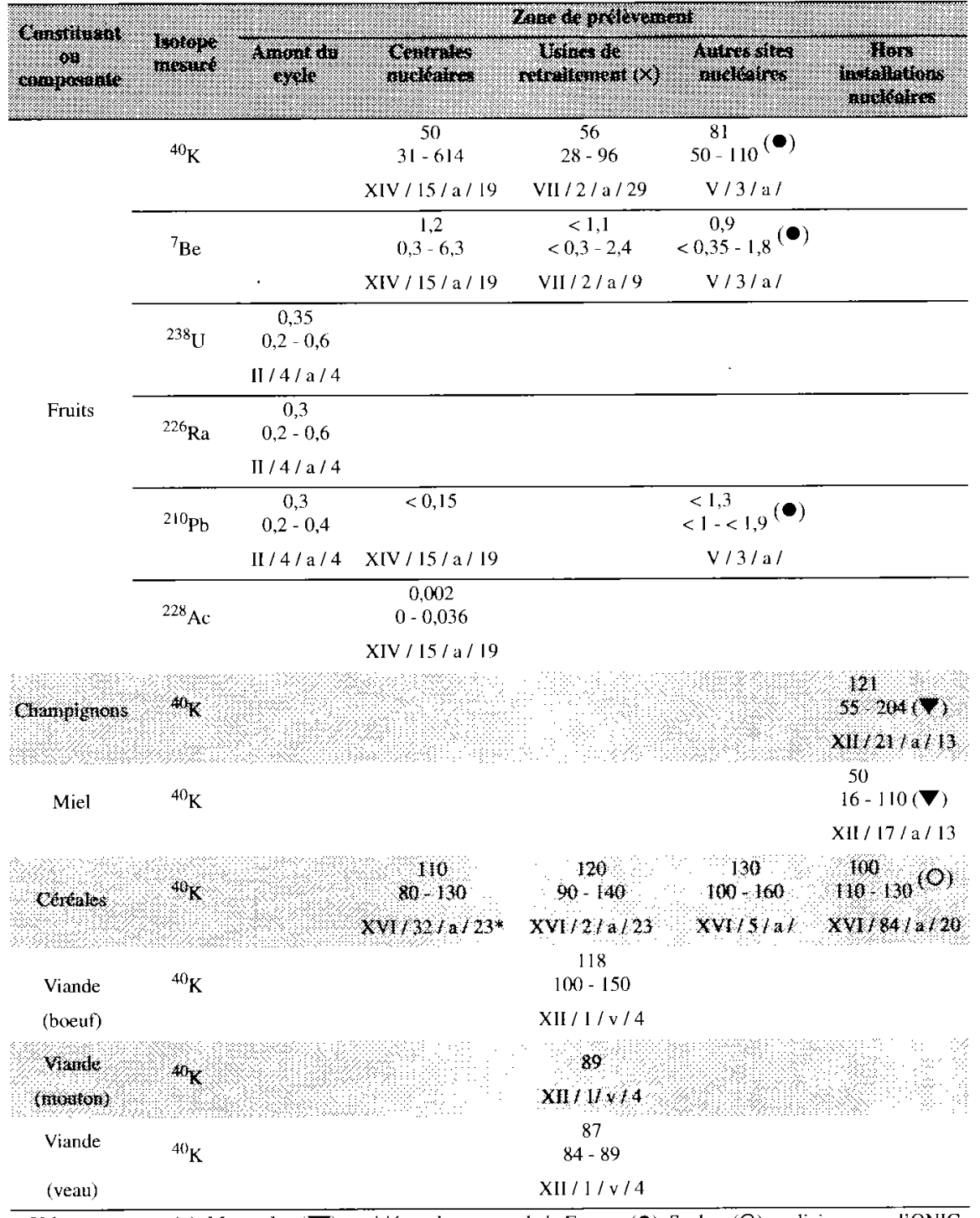

* Valeur moyenne, $(\times)$ Marcoule, ( $\boldsymbol{\nabla})$ moitié nord et centre de la France, $(\bullet)$ Saclay, $(\mathrm{O})$ en liaison avec l'ONIC. 
Des variations de concentrations en isotopes naturels existent entre les différentes espèces végétales qu'il convient de prendre en compte notamment si l'une d'entre elles présente des niveaux d'activité élevés pour certains isotopes naturels et/ou si elle constitue un aliment de base de notre alimentation ${ }^{38}$.

\subsubsection{Poissons, mollusques et crustacés}

Ces denrées constituent, comme les légumes et les fruits, des aliments pour lesquels le délai entre la récolte et la consommation est court, ce qui augmente la possibilité d'impact dosimétrique des isotopes naturels à vie courte tels que le ${ }^{210}$ Po (période $=138$ jours). Cet isotope présente en outre la particularité d'avoir le facteur de dose par ingestion le plus élevé ${ }^{39}$ parmi les radioéléments naturels et d'être par ailleurs bien retenu par certaines espèces marines (crustacés et mollusques) consommées par l'homme (Germain et al., 1992, 1995 ; Ryan et al., 1997).

Pour les poissons d'eau douce, les teneurs en ${ }^{238} \mathrm{U},{ }^{226} \mathrm{Ra}$ et ${ }^{210} \mathrm{~Pb}$ (Tab. XIII) semblent, pour les stations d'échantillonnage situées en amont des rejets des installations des mines d'uranium, légèrement supérieures aux valeurs observées dans l'environnement des centrales ou usines de retraitement ${ }^{40}$. Les doses associées à une consommation annuelle de $5 \mathrm{~kg}$ de poissons frais sont les suivantes:

$$
{ }^{210} \mathrm{~Pb}=4 \mu \mathrm{Sv} ;{ }^{40} \mathrm{~K}=3 \mu \mathrm{Sv} \quad ; \quad{ }^{226} \mathrm{Ra}=2 \mu \mathrm{Sv} ; \quad{ }^{238} \mathrm{U}=0,4 \mu \mathrm{Sv} .
$$

Pour les poissons marins, les données de l'IPSN issues des observatoires marins permettent d'estimer la contribution du ${ }^{210} \mathrm{Po}$ à $15 \mu \mathrm{S} v$ par rapport à une contribution due au ${ }^{40} \mathrm{~K}$ de $4 \mu \mathrm{Sv}$.

Pour les crustacés ou les mollusques filtreurs comme les moules pour lesquels les teneurs en ${ }^{210}$ Po sont élevées en raison des facteurs de concentration plus importants chez ces espèces, la dose annuelle pourrait atteindre $372 \mu \mathrm{Sv}^{41}$.

Des données très ponctuelles existent sur le thorium. Par exemple une étude menée par l'OPRI à proximité de l'usine de la Rochelle sur des moules prélevées

\footnotetext{
${ }^{38}$ La consommation annuelle de pommes de terre représente à elle seule $54 \mathrm{~kg} \mathrm{an}^{-1}$ pour la calégorie des légumes racincs.

${ }^{39} 1,2 \mu S v$ par Becquerel ingéré.

${ }^{40}$ Pour être plus affirmatif, il conviendrait de pouvoir comparer des espèces ayant le même mode d'alimentation el d'êlre certain que les ćchantillons mesurés dans chaque cas correspondaient uniquement à de la chair.

${ }^{41}$ En supposant unc consommation annuelle de $3 \mathrm{~kg}$ de crevettes el de $3 \mathrm{~kg}$ de moules :

moules $41 \mathrm{~Bq} \mathrm{~kg}^{-1}$ frais $\times 3 \mathrm{~kg} \mathrm{an}^{-1} \times 1,2 \times 10^{-6} \mathrm{~Sv} \mathrm{~Bq}^{-1}=148 \mu \mathrm{Sv}$

crevettes $62 \mathrm{~Bq} \mathrm{~kg}^{-1}$ frais $\times 3 \mathrm{~kg} \mathrm{an}^{-1} \times 1,2 \times 10^{-6} \mathrm{~Sv} \mathrm{~Bq}^{-1}=224 \mu \mathrm{Sv}$

Il conviendrait pour une estimation plus prćcise d'évaluer l'effet de la cuisson sur les activités effectivement ingérées.
} 


\section{TABLEAU XIII}

Radioactivité naturelle des poissons, mollusques et erustacés en France ( $\mathrm{Bq} \mathrm{kg}^{-1}$ frais), niveaux non perturbés par l'action de l'homme (notations : $c f$. Fig. 1 et Tab. I).

Natural radioactivity of fish, shellfish and crustaceans in France (Bq $\mathrm{kg}^{-1}$ fresh weight). Levels free from human interference (notations: $c f$. Fig. 1 and Tab. I).

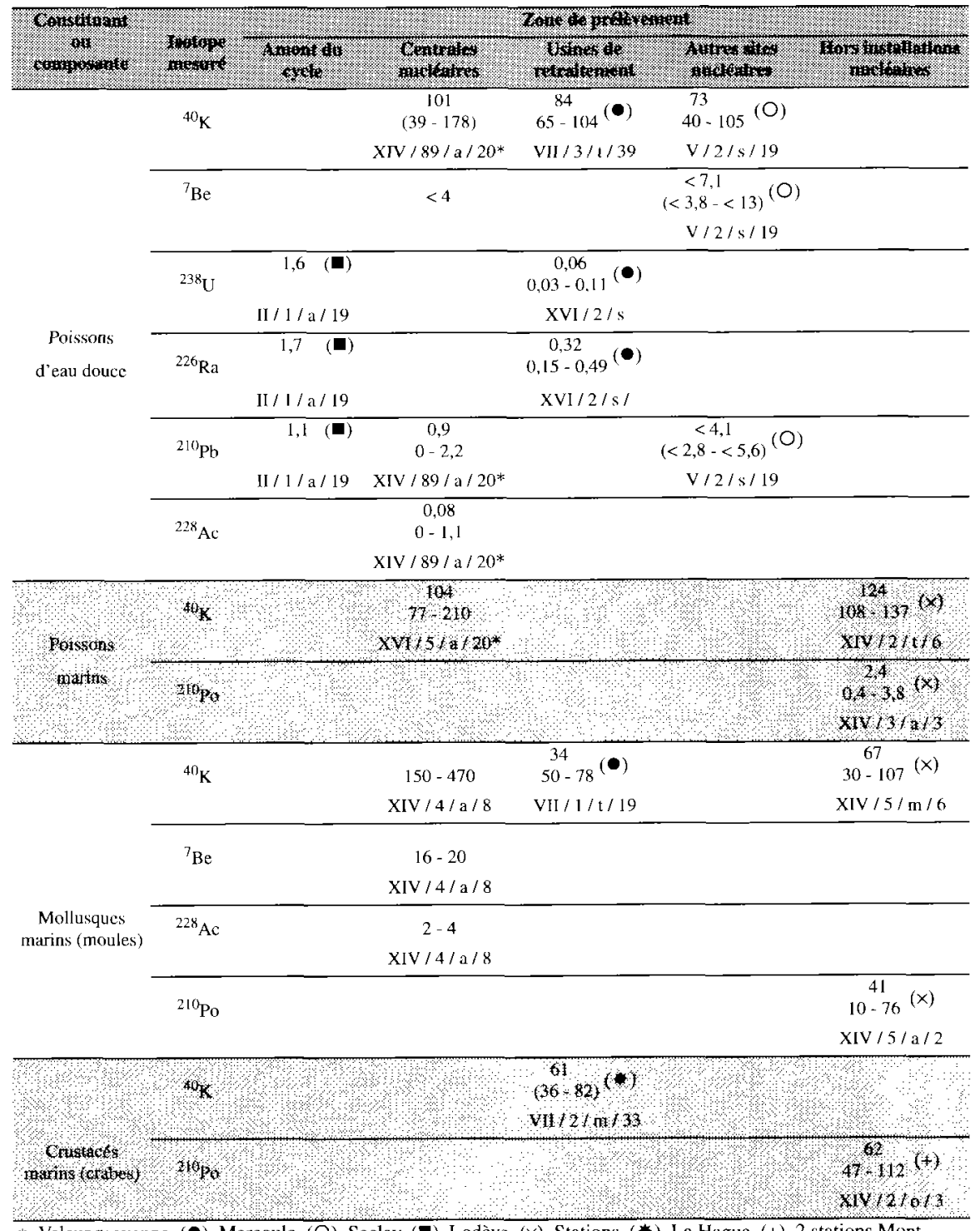

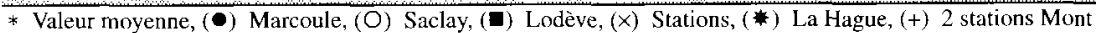
St Michel et Baie de Seine (Crevettes), ( $\times$ ) 3 stations en Manche +2 en baie de Seine. 
en 1989 conduit à des doses annuelles par ingestion dues à cette pratique de $8 \mu \mathrm{Sv}$ pour ${ }^{232} \mathrm{Th}$, de $1,4 \mu \mathrm{Sv}$ pour ${ }^{230} \mathrm{Th}$ et de $2,3 \mu \mathrm{Sv}$ pour ${ }^{228} \mathrm{Th}^{42}$.

\subsection{Radioactivité naturelle des vecteurs et des bio-indicateurs}

\subsubsection{Eaux douces non-filtrées}

Les eaux douces constituent, avec l'atmosphère, le vecteur essentiel de dispersion des éléments dans la biosphère. La connaissance de leur teneur en radioéléments naturels permet, au niveau des stations non perturbées par l'action de l'homme, d'apprécier la variabilité du bruit de fond naturel due aux paramètres (géologie et climatologie) qui agissent sur leur composition et la qualité radioactive de la ressource (Descamps et Foulquier, 1988). Les eaux douces superficielles ou souterraines sont en effet utilisées fréquemment pour l'alimentation en eau potable des populations ou pour l'irrigation (Tab. XIV).

\subsubsection{Végétaux spontanés aquatiques et terrestres}

Le volume de données disponibles est considérable (Tabs. XV et XVI) car les espèces végétales représentatives des milieux aquatiques ou terrestres sont en règle générale d'excellents bio-indicateurs des pollutions véhiculées par les masses d'eau ou d'air.

Quelques remarques générales sur les constituants qui n'entrent pas directement dans notre ration alimentaire sont présentées ci-dessous (Grauby et al., 1984).

Des bioindicateurs comme les mousses aquatiques ou terrestres, les algues, les lichens (Daillant et al., 1996), l'herbe, certaines espèces arbustives permettent de détecter l'impact des rejets d'une installation nucléaire alors que les mêmes mesures réalisées sur des constituants de l'environnement consommés par l'homme ne permettent pas toujours d'identifier la présence de radioéléments artificiels ou naturels exception faite des champignons.

Chez les végétaux terrestres, les radioéléments naturels prédominants sont le ${ }^{40} \mathrm{~K}$, en équilibre avec la teneur en potassium stable des espèces récoltées, et le ${ }^{7} \mathrm{Be}$ qui traduit l'intensité des dépôts secs ou humides récents en provenance de l'atmosphère.

\footnotetext{
${ }^{42}$ Les moules prólevées dans une slation influencée par les rejets de l'usine de La Rochelle montrent des niveaux en ${ }^{232}$ Th, ${ }^{230} \mathrm{Th}$ et ${ }^{228} \mathrm{Th}$ de 15,4 , et $14 \mathrm{~Bq} \mathrm{~kg}{ }^{1}$ frais, à comparer avec des activités de 3,6, 1,8 et 3,2 $\mathrm{Bq} \mathrm{kg}^{-1}$ respectivement mesurćs sur des moules issues d'une station de référence. Consommation annuelle $3 \mathrm{~kg}$ de moules - impact de la pratique pour le ${ }^{232} \mathrm{Th}:(15-3,6) \mathrm{Bq} \mathrm{kg}{ }^{-1}$ Trais $\times 3 \mathrm{~kg} \mathrm{an}^{-1} \times 230 \times 10^{-9} \mathrm{~Sv} \mathrm{~Bq}{ }^{1} \simeq 8 \mu \mathrm{Sv}$.
} 
RADIOACTIVITÉ D'ORIGINE NATURELLE DANS L'ENVIRONNEMENT EN FRANCE

\section{TABL.EAU XIV}

Radioactivité naturelle des eaux brutes en France $\left(\mathrm{Bq}^{-1}\right)$, niveaux non perturbés par l'action de l'homme (notations : $c f$. Fig. 1 et Tab. I).

Natural radioactivity of unprocessed waters in France $\left(\mathrm{Bq} \mathrm{I}^{-1}\right)$. Levels free from human interference (notations: $c f$. Fig. 1 and Tab. I).

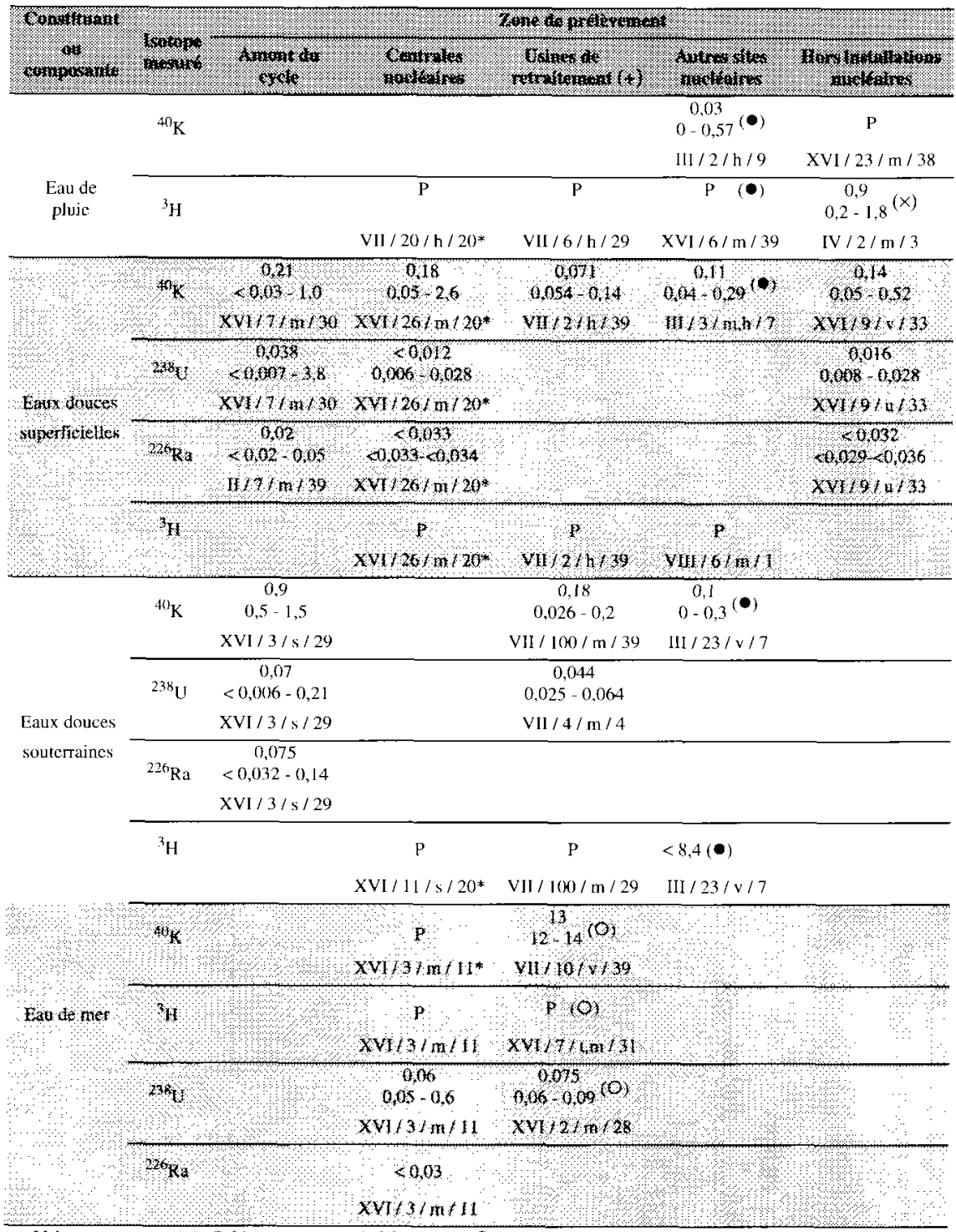

* Valeur moyenne, $(x)$ Orléans - Brest, $(+)$ Marcoule, $(O)$ La Hague, $(\bullet)$ site ANDRA de l'Aube. 
TABLEAU XV

Radioactivité naturelle des végétaux terrestres spontanés en France $\left(\mathrm{Bq} \mathrm{kg}^{-1} \mathrm{sec}\right)$, niveaux non perturbés par l'action de l'homme (notations : $c f$. Fig. 1 et Tab. I).

Natural radioactivity of spontaneous terrestrial plants in France ( $B q \mathrm{~kg}^{-1}$ dry weight). Levels free from human interference (notations: $c f$. Fig. 1 and Tab. I).

\begin{tabular}{|c|c|c|c|c|c|c|}
\hline \multirow{2}{*}{ 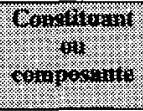 } & \multirow{2}{*}{ Horown } & \multicolumn{5}{|c|}{ trontro prothernow } \\
\hline & & incorifor & noworos & 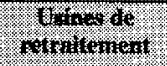 & Hownesing & Howrow \\
\hline \multirow{8}{*}{ Herbe } & \multirow[t]{2}{*}{${ }^{40} \mathrm{~K}$} & & $\begin{array}{c}490 \\
214-1670\end{array}$ & $\begin{array}{c}133 \\
33-260\end{array}$ (口) & $\frac{220}{24-448}(x)$ & $\begin{array}{c}852 \\
213-2168\end{array}$ \\
\hline & & & $\mathrm{XIV} / 19 / \mathrm{a} / 23$ & $\mathrm{VII} / 10 / \mathrm{m} / 29$ & $\mathrm{~V} / 6 / \mathrm{m} /$ & $\mathrm{XIV} / 20 / \mathrm{a} / 6$ \\
\hline & \multirow[t]{2}{*}{${ }^{7} \mathrm{Be}$} & & $\begin{array}{c}183 \\
10-550\end{array}$ & $\begin{array}{c}87 \\
(4-427)\end{array}(\square)$ & $11-326(x)$ & $\begin{array}{c}262 \\
25-1064\end{array}$ \\
\hline & & & $\mathrm{XIV} / 18 / \mathrm{a} / 23$ & $\mathrm{VII} / 10 / \mathrm{m} / 29$ & $\mathrm{~V} / 6 / \mathrm{m} /$ & XIV / $20 / a / 6$ \\
\hline & \multirow[t]{2}{*}{${ }^{228} \mathrm{Ac}$} & & $\begin{array}{c}3 \\
0-21\end{array}$ & & & $\begin{array}{c}0,5 \\
0-4,1\end{array}$ \\
\hline & & & $\mathrm{XIV} / 18 / \mathrm{a} / 23$ & & & $\mathrm{X \Gamma V} / 20 / \mathrm{a} / 6$ \\
\hline & \multirow[t]{2}{*}{${ }^{210} \mathrm{~Pb}$} & & $\begin{array}{c}14 \\
7,7-22\end{array}$ & & & \\
\hline & & & $\mathrm{XIV} / 18 / \mathrm{a} / 23$ & & & \\
\hline \multirow{9}{*}{ 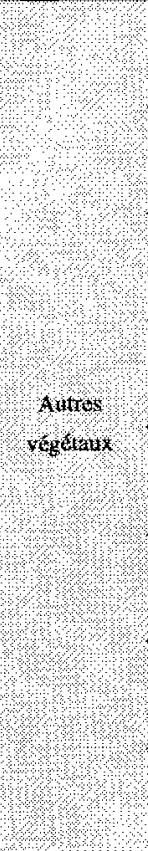 } & +1k & & $\begin{array}{l}28,3,0) \\
\times 1 \% / 6,4,10\end{array}$ & $\begin{array}{l}204 \\
(187) 218) \\
11 / 2 / s)\end{array}$ & 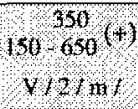 & 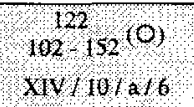 \\
\hline & 18 & & (4) 845 (0) & $\left(1872_{18)}(6)\right.$ & $80,400(t)$ & 1096,70 \\
\hline & & & $\mathrm{XN} / 63 / \mathrm{N} 10$ & $11 / 2,1 / 9$ & $\sqrt{ } / 2 \mathrm{nl}$ & $x+10 / 9,6$ \\
\hline & 30 & $\begin{array}{l}2,1) \\
\text { मा }\end{array}$ & 8 & 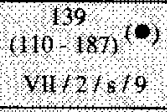 & & \\
\hline & $\begin{array}{l}4 \mathrm{Ma} \\
\mathrm{H}\end{array}$ & $\begin{array}{l}2,84) \\
1 / 10\end{array}$ & & & & में' \\
\hline & स4, & & मे? & 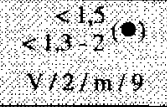 & & \\
\hline & म19 & $\begin{array}{l}\text { 6/f } \\
\mathrm{H} / \mathrm{l}\end{array}$ & 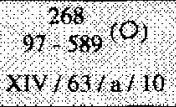 & 4 & & 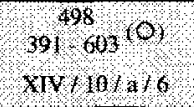 \\
\hline & मf & & & औ? & & 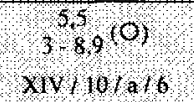 \\
\hline & $2 / 2 \mathrm{pb}$ & & & 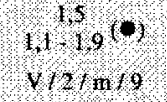 & & मे? \\
\hline
\end{tabular}

(D) Foin, ( $\times$ ) Fontenay-aux-Roses, (@) Thym à Marcoule, ( $\square$ ) Marcoule, (O) Mousses terrestres, (+) Thym à Cadarache. 


\section{TABLEAU XVI}

Radioactivité naturelle des végétaux aquatiques spontanés en France $\left(\mathrm{Bq} \mathrm{kg}^{-1}\right.$ sec), niveaux non perturbés par l'action de l'homme (notations : $c f$. Fig. 1 et Tab. I).

Natural radioactivity of spontaneous aquatic plants in France $\left(\mathrm{Bq} \mathrm{kg}^{-1} \mathrm{dry}\right.$ weight). Levels free from human interference (notations: $c f$. Fig. 1 and Tab. I).

\begin{tabular}{|c|c|c|c|c|c|c|}
\hline \multirow{2}{*}{ 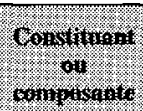 } & \multirow[b]{2}{*}{ rotord } & \multicolumn{4}{|c|}{ 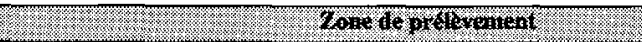 } & \multirow[b]{2}{*}{ Wownowhor } \\
\hline & & 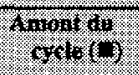 & 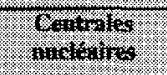 & Wring do & 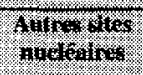 & \\
\hline \multirow{15}{*}{$\begin{array}{c}\text { Plantes } \\
\text { d'eau douce }\end{array}$} & \multirow[t]{2}{*}{${ }^{40} \mathrm{~K}$} & & $\begin{array}{c}990 \\
331-1721\end{array}$ & $\begin{array}{c}632 \\
(267-1020)\end{array}$ & $146-616(x)$ & $\begin{array}{c}1076 \\
532-2007\end{array}$ \\
\hline & & & $\mathrm{XIV} / 10 / \mathrm{a} / 21$ & $\mathrm{VII} / 3 / \mathrm{t} / 29$ & $\mathrm{~V} / \mathrm{l} / \mathrm{t} /$ & $\mathrm{XIV} / 7 / \mathrm{a} / 20$ \\
\hline & \multirow[t]{2}{*}{${ }^{7} \mathrm{Be}$} & & $\begin{array}{c}55 \\
19-153\end{array}$ & $\begin{array}{c}58 \\
(20-98)\end{array}$ & $52-174(x)$ & $13-189(+)$ \\
\hline & & & $X I V / 10 / a / 21$ & $\mathrm{VII} / 3 / \mathrm{t} / 29$ & $V / 1 / t /$ & $\mathrm{XIV} / 7 / \mathrm{a} / 20$ \\
\hline & \multirow[t]{2}{*}{${ }^{238} \mathrm{U}$} & $\begin{array}{c}0,8 \\
0,4-2,2(\mathbf{\square})\end{array}$ & & & & \\
\hline & & II $/ 6 / \mathrm{a} / 19$ & & & & \\
\hline & \multirow[t]{2}{*}{${ }^{226} \mathrm{Ra}$} & $\begin{array}{c}1,8 \\
0,4-3,7\end{array}$ & & & & \\
\hline & & II/ $/ 6 / a / 19$ & & & & \\
\hline & \multirow[t]{2}{*}{${ }^{214} \mathrm{~Pb}$} & \multirow{2}{*}{\multicolumn{4}{|c|}{$\begin{array}{c}19,6 \\
<5,4-47 \\
\text { VII } / 3 / t / 29\end{array}$}} & \\
\hline & & & & & & \\
\hline & \multirow[t]{2}{*}{${ }^{210} \mathrm{~Pb}$} & $\begin{array}{c}3,2 \\
0,3-7,5\end{array}$ & $\begin{array}{c}24 \\
12-42\end{array}$ & & & ${ }_{16-29}^{21}(+)$ \\
\hline & & II $/ 6 / \mathrm{a} /$ & $\mathrm{XIV} / 10 / \mathrm{a} / 21$ & & & $\mathrm{XIV} / 7 / \mathrm{a} / 20$ \\
\hline & \multirow[t]{2}{*}{${ }^{228} \mathrm{Ac}$} & & $\begin{array}{l}33 \\
3-170\end{array}(\bullet)$ & & & $0_{0-57}^{20}(+)$ \\
\hline & & & $\mathrm{XIV} / 10 / \mathrm{a} / 21$ & & & $\mathrm{XIV} / 7 / \mathrm{a} / 20$ \\
\hline & ${ }^{212} \mathrm{~Pb}$ & \multicolumn{4}{|c|}{$\begin{array}{c}14 \\
4,3-15 \\
\text { VII } / 3 / t / 29\end{array}$} & \\
\hline \multirow{9}{*}{$\begin{array}{l}\text { Algtes } \\
\text { (Fucas) }\end{array}$} & \multirow[t]{2}{*}{${ }^{40} \mathrm{~K}$} & & $871809(0)$ & $522+1370$ & & $71-1990(0)$ \\
\hline & & & $x \sim 1 / 4, \alpha$ & $x \operatorname{IV} 12 / \mathrm{m} / 2$ & & $\mathrm{xiv} / \mathrm{s} / \mathrm{m} / 6$ \\
\hline & \multirow[t]{2}{*}{$\mathrm{Be}_{\mathrm{B}}$} & & $58-22(0)$ & $\begin{array}{r}26 \\
9 \times 31\end{array}$ & & $<64(1)$ \\
\hline & & & $\mathrm{xW} / 4 / a / 8$ & $X \mid \vee t 2 t \mathrm{~m} / 25$ & & $\mathrm{X} Y \mathrm{~A} / \mathrm{m} / 6$ \\
\hline & \multirow[t]{2}{*}{$21 \mathrm{pb}$} & & $16-19(0)$ & & & \\
\hline & & & $\mathrm{XIV} / 4 / 8 / 8$ & & & ४। \\
\hline & \multirow[t]{2}{*}{$27 \mathrm{Ac}$} & & $2-10(0)$ & $\begin{array}{r}92 \\
5.12 \mathrm{z}\end{array}$ & & सा 6 (1) \\
\hline & & & $\mathrm{XrV} / 4 f \mathrm{a} / \mathrm{8}$ & $\mathrm{XIV} / 2+\mathrm{a} / 2 \mathrm{~s}$ & & $\mathrm{xU} / \mathrm{s}, \mathrm{fo}$ \\
\hline & 2100 & & & & & 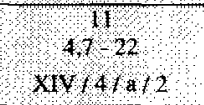 \\
\hline
\end{tabular}

(ם) roseaux ajoncs, $(\bullet)$ Centrales EDF continentales (mousses aquatiques), $(x)$ Grenoble, $(\square)$ Observatoire marin, (O) Centrales EDF bord de mer, (+) stations à l'amont de tout rejet sur les cours d'eau (mousses aquatiques). 
L'intérêt comme bio-indicateur des mousses terrestres est mis en évidence, par exemple, par leur teneur en ${ }^{210} \mathrm{~Pb}$ pour des échantillons récoltés à proximité de centrales $\left(268 \mathrm{~Bq} \mathrm{~kg}^{-1} \mathrm{sec}\right)$ ou dans des zones dépourvues d'installations nucléaires (498 $\mathrm{Bq} \mathrm{kg}^{-1} \mathrm{sec}$ ) alors que du foin récolté en zone uranifère ne présente qu'une activité de $4 \mathrm{~Bq} \mathrm{~kg}^{-1} \mathrm{sec}$.

Pour les plantes aquatiques, la prédominance du ${ }^{40} \mathrm{~K}$ est encore plus accentuée notamment chez les algues (Lambrechts et al., 1992 ; Patti et al., 1990). La réponse spécifique des espèces vis-à-vis de la rétention des radioéléments naturels est à nouveau illustrée par les écarts de concentration en ${ }^{210} \mathrm{~Pb}$ d'un facteur 7 à 8 existant entre les roseaux et les ajoncs d'une part et les mousses aquatiques d'autre part.

Des différences de concentration pour un isotope naturel donné peuvent aussi apparaître entre deux espèces voisines de champignons (Calmet et al., 1998b) ou d'algues. Une attention particulière doit donc être consacrée au choix des espèces végétales à échantillonner. À défaut, la variabilité du bruit de fond naturel et/ou l'impact additionnel dû à une pratique impliquant des isotopes naturels pourraient être mal appréciés.

\subsection{Cas du carbone-14}

En dépit du fait qu'il est le principal radioélément d'origine cosmique qui contribue aux doses délivrées aux populations, les données concernant le ${ }^{14} \mathrm{C}$ au voisinage des installations nucléaires françaises sont peu nombreuses. En outre, déterminer les concentrations caractérisant le bruit de fond naturel dans le but d'évaluer l'incidence d'une pratique impose de mesurer l'activité en ${ }^{14} \mathrm{C}$ contenu par kilogramme de carbone stable présent dans chaque échantillon. En effet, produit naturellement ou artificiellement, transformé en ${ }^{14} \mathrm{CO}_{2}$, il se mélange au gaz carbonique de l'air ${ }^{12} \mathrm{CO}_{2}$ et s'intègre au cycle biologique. Pour tout végétal en relation avec l'atmosphère, le bruit de fond naturel en ${ }^{14} \mathrm{C}$ (Fig. 4) se caractérise actuellement ${ }^{43}$ par une activité de $226 \mathrm{~Bq}$ de ${ }^{14} \mathrm{C}$ par kilogramme de carbone. La contribution actuelle due aux anciennes explosions atomiques aériennes représentait en 1997 un apport supplémentaire de 25 à $30 \mathrm{~Bq}$ de ${ }^{14} \mathrm{C}$ par kilogramme de carbone stable (Fournier, 1998).

Un rejet artificiel en ${ }^{14} \mathrm{C}$ peut donc entraîner localement un dépassement de la valeur de $226+(25$ à 30$)=251$ à $256 \mathrm{~Bq}$ de ${ }^{14} \mathrm{C}$ par kilogramme de carbone stable qui caractérise en 1997 tout végétal en contact avec l'atmosphère. Par contre,

\footnotetext{
${ }^{43}$ Dans les années 60 , cette teneur atlcignail en France plus de 400 becqucrel de ${ }^{14} \mathrm{C}$ par kilogramme de carbone du fait du nombre d'explosions d'engins nucléaires réalisées à cette époque dans l'atmosphère de l'hémisphère nord.
} 


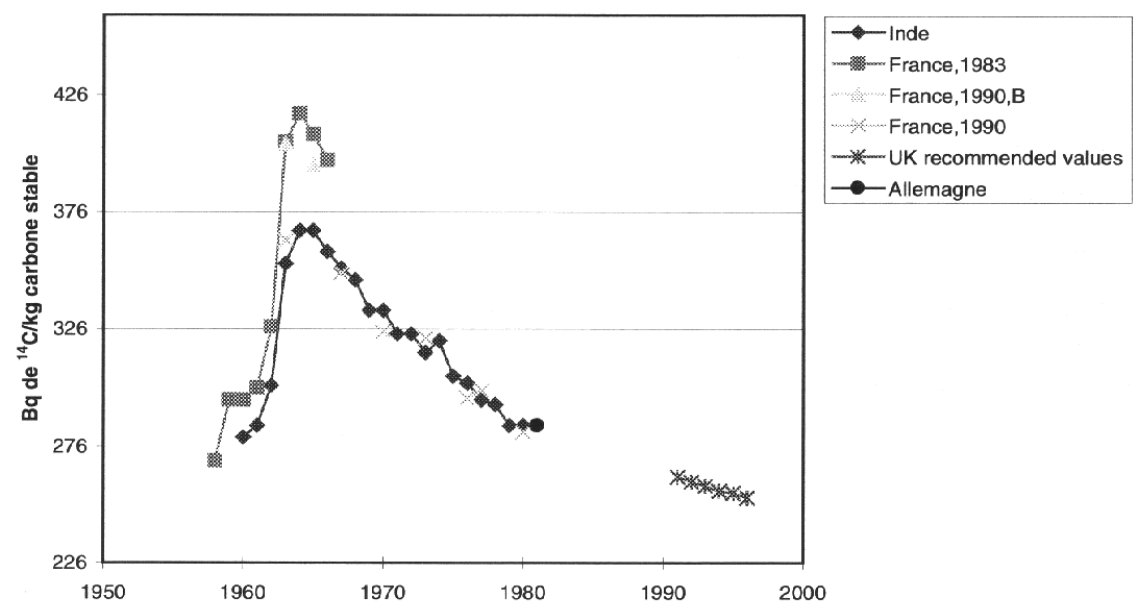

Figure 4 - Variation de la teneur en ${ }^{14} \mathrm{C}$ de végétaux chlorophylliens en Europe de l'ouest de 1958 à 1998. ${ }^{14} \mathrm{C}$ content variations in chlorophyll contents of plants of Western Europe from 1958 to 1998.

lorsque le végétal meurt et ne se met plus en équilibre à travers les processus de la photosynthèse avec l'atmosphère, sa teneur en ${ }^{14} \mathrm{C}$ par gramme de carbone décroît ${ }^{44}$.

L'expression des résultats en $\mathrm{Bq}$ de ${ }^{14} \mathrm{C}$ exprimés en masse ou en volume, reste indispensable pour estimer les expositions sous la forme de doses. Parmi les différents radionucléides cosmogéniques, le carbone-14 est celui qui contribue le plus à la dose reçue par les populations. Sur la base d'une production annuelle de $1 \mathrm{PBq}$ et une activité spécifique de $230 \mathrm{~Bq} \mathrm{~kg}^{-1}$ de carbone dans la matière organique vivante, la dose efficace annuelle est estimée à $12 \mu \mathrm{Sv}$ (UNSCEAR, 1993).

Mais la connaissance des activités en ${ }^{14} \mathrm{C}$ exprimées par kilogramme de carbone stable d'un échantillon permet seule de savoir si, en tenant compte de l'origine de l'échantillon mesuré ${ }^{45}$, son activité est le reflet du bruit de fond naturel ou si au contraire il traduit l'impact d'une pratique.

Ainsi, lors de la comparaison des résultats obtenus par les modèles avec ceux issus de mesures sur des échantillons de végétaux, prélevés dans l'environnement

\footnotetext{
${ }^{44}$ C'est le principe de la datation par le carbone-14.

${ }^{45}$ Pour des constituants de l'environnement dont la teneur en carbone n'est pas obligatoirement liée uniquement au $\mathrm{CO}_{2}$ présent dans l'atmosphère (sols, sédiment, eaux, animaux), la valeur de $250-256 \mathrm{~Bq}$ de ${ }^{14} \mathrm{C}$ par kilogramme de carbone stable ne peut servir de référence pour évaluer l'impact d'une pratique.
} 
du plateau de La Hague entre 1996 et 1998, le Groupe radioécologie nord-Cotentin (GRNC) a relevé des activités de ${ }^{14} \mathrm{C}$ de l'ordre de 2 à 3 fois le bruit de fond naturel, comprises entre 500 et $900 \mathrm{~Bq} \mathrm{~kg}^{-1}$ de carbone. Dans ce contexte et pour un scenario particulier, celui des « agriculteurs vivant à Pont Durand en 1996 », le GRNC a établi que le ${ }^{14} \mathrm{C}$ contriburait à prés du tiers de la dose efficace annuelle, soit $18 \mu \mathrm{Sv}$ sur $59 \mu \mathrm{Sv}$ (GRNC, 1999).

\section{Conclusion}

Cette enquête est la première de ce genre dans le domaine de la radioactivité naturelle de par son approche multipartenaires. Dix organismes publics et associations ont participé à ce travail collectif de synthèse des données initialement éparses (Annexe). Cette synthèse a permis de dresser un premier état des niveaux de radioactivité des principaux radionucléides artificiels en fonction des caractéristiques environnementales du territoire métropolitain. Ce travail mérite certainement d'être approfondi.

Les différences d'objectifs des organismes participants et donc les différences entre les approches et les méthodologies d'acquisition des données qui en résultent, sont limitantes pour une interprétation complète des niveaux d'activité.

Cette enquête montre la difficulté actuelle de dresser en France un bilan exhaustif des connaissances acquises concernant les caractéristiques du bruit de fond radioactif d'origine naturelle dans l'environnement. Or, la radioactivité naturelle constitue une des composantes essentielles de l'irradiation des populations. En outre, sans cette connaissance de base, il est impossible d'évaluer la part de l'impact des pratiques susceptibles de modifier la distribution des radioéléments d'origine tellurique ou cosmique dans notre environnement.

Il serait donc souhaitable que soit mise en place au niveau national une base de données informatisées tenant compte des résultats acquis actuels, de l'évolution de la réglementation (JOCE, 1996) et des réflexions en cours ${ }^{46}$.

L'attente de cet objectif requiert de :

- sélectionner parmi les stations où sont réalisées actuellement des mesures, des stations de référence caractérisées par l'absence de toute modification des niveaux d'activités naturelles liée à une pratique,

de disposer, à ces stations, de séries d'observations chronologiques à long terme afin d'apprécier au mieux la variabilité spatio-temporelle des rayonnements et des radionucléides d'origine naturelle,

${ }^{46}$ Document préparatoire “ Exposures from natural sources » 48 ${ }^{\mathrm{e}}$ session de l'UNSCEAR. Vienne I2-16 Avril 1999. 
- privilégier la mesure des rayonnements et des isotopes qui contribuent le plus aux doses délivrées aux populations en raison de leurs caractéristiques propres, du mode de vie des populations, et des pratiques actuelles ou passées,

- utiliser des protocoles communs d'échantillonnage, de conditionnement des échantillons, d'analyses et de traitement des résultats qui garantissent la validité des interprétations basées sur des données recueillies à des époques, en des lieux et sur des constituants différents,

- de confier à un organisme au niveau national la coordination des travaux entrepris par les différents intervenants et la gestion de la base de données proposée.

Une réelle amélioration de l'évaluation des risques liés aux applications de l'énergie nucléaire peut résulter d'une diversification des organismes effectuant des mesures et d'une multiplication des contrôles réalisés ${ }^{47}$. Mais aujourd'hui en France, dans le domaine de la radioactivité naturelle, les efforts consentis manquent de coordination et d'orientations prioritaires.

À ce titre, les mesures des ${ }^{238} \mathrm{U}$ et ${ }^{232} \mathrm{Th}$ précurseurs des ${ }^{226}$ et ${ }^{228} \mathrm{Ra}$, des descendants solides émetteurs alpha du radon (par la mesure des EAP du ${ }^{222} \mathrm{Rn}$ et $\left.{ }^{220} \mathrm{Rn}\right)$, des émetteurs gamma post émanation $\left({ }^{214} \mathrm{~Pb},{ }^{214} \mathrm{Bi},{ }^{212} \mathrm{~Pb}\right), \mathrm{du}{ }^{210} \mathrm{~Pb}$, ${ }^{210}$ Po et du ${ }^{14} \mathrm{C}$ doivent être privilégiées.

Remerciements. Les rédacteurs expriment leurs remerciements à tous ceux qui ont participé à cette enquête en transmettant des données et qui n'apparaissent pas en tant que cosignataires de cette publication.

\section{Annexe}

Coordonnées des organismes effectuant des mesures de radioactivité dans l'environnement en France et publiant des informations sur les niveaux de radioactivité en France.

\section{- ALGADE}

1 avenue du Brugeaud, BP 46, 87250 Bessines-sur-Gartempe tél. : 05.55.60.50.00, fax : 05.55.60.50.59

e-mail : algade@algade.com, site internet : www.algade.com

- Brochure Algade radioprotection et instrumentation.

\footnotetext{
${ }^{47}$ Actuellement, les données existantes sur la radioactivitê naturelle dans l'environnement à l'extéricur des bâtiments représentent plusieurs millions d'informations.
} 


\section{- ANDRA}

1-7 rue Jean Monnet, Parc de la Croix Blanche, 92298 Chatenay-Malabry Cedex

tél. : 01.46.11.80.00, fax : 01.46.11.81.00

site internet: www.andra.fr

- Plaquettes trimestrielles « Centre de la Manche - La surveillance de l'Environnement », Centre de l'Aube - La surveillance de l'Environnement »,

- Plaquette «Centre de la Manche »,

- Cassettes : « 25 ans d'histoire du Centre de la Manche », «Le Centre de l'Aube ».

- Association pour le contrôle de la radioactivité dans l'ouest (ACRO)

138 rue de l’Église, 14200 Hérouville St Clair

tél. : 02.31.94.35.34, fax : 02.31.94.85.31

site internet : www.acro.fr.st

- Revue trimestrielle « L'ACROnique du nucléaire ».

- Groupe BRGM

3 avenue Claude Guillemin, BP 6009, 45060 Orléans Cedex 2

tél. : 02.38.64.34.34, fax : 02.38.64.35.18

site internet : www.brgm.fr

- Plaquette Antea,

- Rapport BRGM R40465.

\section{- CEA/DCS/SHSP}

CEA/FAR, BP 6, 92265 Fontenay-aux-Roses Cedex

tél. 01.46.54.74.96, fax : 01.46.54.81.78

site internet: www.cea.fr

- «Contrôle des rejets et surveillance de l'environnement des centres CEA Bilan annuel », Éditions 1996-1997-1998.

Sous la responsabilité des centres CEA :

- Établissement de Bruyères Le Châtel, plaquette mensuelle «surveillance de l'environnement ",

- CEA/Grenoble, plaquette mensuelle « Lettre d'information semestrielle de l'Environnement »,

- CEA/Cadarache, plaquette trimestrielle « Carte sur table » : bilan des rejets et de la surveillance de l'environnement»,

- CEA/Saclay, plaquette semestrielle «CEA/Saclay, l'environnement ». 
- CENTRE d'essais et de contrôle de la radioactivité

\section{CRECEP}

1 voie de Bouvray, 94310 Orly

tél. : 01.48.90.93.87, fax : 01.48.90.50.61

\section{- CNRS/LSCE}

Domaine du CNRS, avenue de la Terrasse, 91198 Gif-sur-Yvette Cedex tél. : 01.69.82.35.35, fax : 01.69.82.35.68

site internet : www.cnrs.fr

- COGEMA/Branche uranium/Direction des opérations minières/SEPA
BP 71, 87250 Bessines-sur-Gartempe
tél. : 05.55.76.64.40, fax : 05.55.76.64.59
site internet: www.cogema.fr
- La lettre de l'Ecarpiere - édition semestrielle,
- La lettre de la Crouzille - édition trimestrielle,
- La lettre de Lodève - édition trimestrielle,
- La lettre du Forez - édition annuelle.

\section{- COGEMA La Hague}

50444 Beaumont-Hague Cedex

tél. : 02.33.02.62.02, fax : 02.33.02.76.07

- Rapport Environnement Cogema La Hague 1997,

- Plaquette périodique surveillance de l'environnement.

\section{- COGEMA Marcoule}

BP 170, 30206 Bagnols-sur-Cèze Cedex

tél. : 04.66.79.53.45, fax. : 04.66.79.57.01

- Rapport Environnement Cogema, Marcoule 1997,

- Plaquette mensuelle, Surveillance de l'environnement,

- Cassette « le contrôle de l'environnement d'un site nucléaire », COGEMA/CEA VALRHO/SOCODEI. 
- CRII-RAD : Commission de recherche et information indépendantes sur la radioactivité

471 avenue Victor Hugo, 26000 Valence

tél. : 04.75.41.82.50, fax : 04.75.81.26.48

e-mail : contact@criirad.com

- Revue trimestrielle « Le trait d'union »,

- Fiches thématiques : "La contamination des champignons », «Le radon », «Tchernobyl : contamination du territoire français ».

- Direction générale de la concurrence, consommation et répression des fraudes (DGCCRF)

* Ministère de l'économie et des finances et de l'industrie 59 boulevard Vincent Auriol, 75703 Paris Cedex 13 tél. : 01.44.97.30.74

* Laboratoire interrégional de la répression des fraudes de Lille 369 rue Jules Guesde, BP 39, 59651 Villeneuve Cedex tél. : 03.20.79.95.95, Fax : 03.20.47.28.23

- Brochure Laboratoires 1997.

- EDF/DEPT/DSRE

6 rue Ampère, BP 114, 92203 Saint Denis Cedex 1

tél. : 01.43.69.31.27, fax : 01.43.69.45.71

site internet: www.edf.fr

36.14 : MAGNUC-SIM-EDFME

- Brochure « Nucléaire et Environnement »- Année 1997.

- Institut de radioprotection et de sûreté nucléaire

Mission communication

tél. : 01.58.35.86.38, fax : 01.58.35.84.51

77-83 avenue du général de Gaulle, 92140 Clamart

BP 17, 92262 Fontenay-aux-Roses Cedex

site internet : www.irsn.fr

- Lyonnaise des eaux

Centre international de recherches sur l'eau et l'environnement 38 rue du président Wilson, 78230 Le Pecq

tél. : 01.34.80.23.76, fax : 01.30.53.62.09 site internet: www.lyonnaise.des.eaux.fr

- Gestion station d'alerte sur la radioactivité des eaux de la Seine en aval de la Centrale de Nogent. 
- Marine nationale/Groupe d'études atomiques

BP 34, 50115 Cherbourg naval

tél. : 02.33.92.65.97, fax : 02.33.94.24.00

- Plaquette semestrielle « surveillance radiologique de l'environnement des ports de Cherbourg, Brest et Toulon »,

- Cassette «Réseau surveillance nucléaire marine».

\section{- SGS/Laboratoire Crepin}

2 rue Dugey Trouin, BP 1282, 76178 Rouen Cedex

tél. : 02.35.07.91.80, fax : 02.35.07.91.93

- Dossier de présentation Environnement.

\section{- SUBATECH - École des Mines de Nantes}

4 rue Alfred Kastler, La Chantrerie, BP 20722, 44307 Nantes Cedex 3

tél. : 02.51.85.81.00, fax : 02.51.85.84.79

site internet: www-subatech.in2p3.fr

- Brochure SMART/SUBATECH

\section{- Université de Franche Comté}

Institut des sciences et techniques de l'environnement (ISTE), BP 7 1427, 25211 Montbéliard Cedex

tél. : 03.81.99.46.92, fax : 03.81.99.46.85

- Plaquette activités ISTE.

\section{RÉFÉRENCES}

AIEA (1992) Statistical treatment of data on Environmental Isotopes in Precipitation, Technical reports series $\mathrm{N}^{\circ} 331$, Vienna, Austria.

AIEA (1994) Handbook of parameter Values for the Prediction of radionucleide transfer in Temperate Environments, Technical reports serics No 364 , Vienna, Austria.

Aigueperse J., Chalabreysse J., Coulon R., Grauby A., Uzzan G. (1981) Impact radiologique des rejets atmosphériques d'une centrale au charbon, pp. 195-214, Proceedings of a Symposium on Health Impacts of Different Sources of Energy, Nashville (USA), 22-26 juin 1981, 701 p.

ANDRA (1998) Agence nationale de gestion des déchets radioactifs, Inventaire national des déchets radioactifs, Chatenay-Malabry, France.

Belot Y., Roy M., Métivier M. (1996) Le tritium de l'environnement à l'homme, Coll. IPSN. Éditions de Physique, les Ulis, France.

Bernhard S., Vauzelle Y., Zettwoog P. (1995) Measurement of the radiological impact of environmental radon 222 releases from heaps of solid wastes from the mineral industry pp. 2024, IRPA endorsed Int. Conf. on Radioprotection and Radioactive Waste Management in the Mining and Mineral Industries, Johannesburg, South Africa. 
Calmet D., Bouisset P., Barker E. (1998a) Comportement du béryllium 7 atmosphérique, Journées SFRP : Les irradiations naturelles en France, Poitiers, France, 17-18 mars 1998.

Calmet D., Boursier B., Bouisset P., Guiard A., Barker E. (1998b) Mushrooms as reference material for intercomparison exercises and as bioindicators of radiocesium deposition in soil (France and Central European Countries), Appl. Rad. Isot. 49 (1-2), 19-28.

Caput C., Guenot J., Belot Y., Bourdeau F. (1981) Méthodologie de la mesure des émissions et des immissions de carbone 14 rejeté dans l'atmosphère par les centralles nucléaires françaises, $A I E A$ Colloque international sur les migrations dans l'environnement terrestre des radionucléides de longue période provenant du cycle du combustible nucléaire, Knoxville (USA) 2731 juillet 1981.

Daillant O., Kirchner G., Jacquiot L., Lecomte A., Tillier C., van Haluwyn C. (1996) Recherche d'éléments radioactifs naturels dans quelques lichens foliacés, Pollution Atmosphérique oct/nov, 65-79.

Descamps B., Baudin-Jaulent Y. (1987) Étude radioécologique du complexe minier de Lodève (France), 1981-1985, Rapport CEA - R-5409, 98 p.

Descamps B., Foulquier L. (1988) Natural radioactivity in the principal constituents of French river ecosystems, $I V^{e}$ International Symposium on the natural radiation environment, Lisbonne, Portugal, 7-11 décembre 1987; Rad. Prot. Dosim. 24 (1-4), 143-147.

EC (1998) European commission D.G. XI, Environmental Radioactivity in the European Community 1993, Rapport EUR 17714, 1998, 82 p.

Foulquier L., Pally M. (1982) Données sur la teneur en tritium lié de poissons des grands fleuves français, Journées de l'Union Internationale des Radioécologistes, Wageningen, Hollande, 2830 juin $1982,23 \mathrm{p}$.

Fournier M. (1998) Carbone 14 et tritium dans l'environnement. Journées SFRP : Les irradiations naturelles en France, Poitiers, France, 17-18 mars 1998.

Germain P., Leclerc G., Simon S. (1992) Distribution of ${ }^{210}$ Po in Mytilus Edulis and Fucus Vesiculosus along the channel coast of France ; Influence of industrial releases in the seine river and estuary, Rad. Prot. Dosim. 45 (1-4), 257-260.

Germain P., Leclerc G., Simon S. (1995) Transfert of polanium-210 into Mytilus edulis (L) and Fucus vericulosus (L) from Baie de Seine (Channel coast of France), Sci. Tot. Environm. 164, $109-123$.

Gontier G. (1998) Le potassium 40 dans l'environnement, Journées SFRP : les irradiations naturelles en France, Poitiers, 17-18 Mars 1998.

Grauby A., Bourdeau F., Ottavi L., Hugon J. (1984) Mesures de radioactivité dans l'environnement : résultats et commentaires, Congrès sur les données actuelles sur la radioactivité naturelle, Monte-Carlo, 5-7 novembre 1984.

GRNC (1999) Estimation des doses et du risque de leucémie associée, Rapport GRNC, Vol, 4, 365 p.

Guerere M., Tisse C., Pelleau Y., Picat P., Bernard J., Quinault J.M., Santoni P., Cartier Y., Feuerstein J. (1989) Niveaux de radioactivité de la ration alimentaire d'un citadin de la région PACA deux ans après Tchernobyl. Enseignements en terme de qualité et de sécurité des aliments, Séminaire sur le transfert de la radioactivité au cours des transformations agro-alimentaires et des préparations culinaires, Cadarache, 18-21 septembre 1989.

ICRP Publication 65 (1993) International commission on radiological protection against radon-222 at home and at work, Ann. ICRP 22 (2), Pergamon Press, Oxford, UK.

Jammet H. (1984) Importance relative de l'irradiation naturelle dans l'ensemble des expositions humaines, Congrès sur les données actuelles sur la radioactivité naturelle, Monte-Carlo, 5-7 novembre 1984 . 
JOCE (1996) Journal officiel des communautés européennes, Directive 96/29, Euratom du conseil du 13 mai 1996, fixant les normes de base relatives à la protection sanitaire de la population et des travailleurs contre les dangers résultant des rayonnements ionisants, $\mathrm{N}^{\circ} \mathrm{L} 159$ du 29 juin 1996.

Lambrechts A., Foulquier L., Garnier-Laplace J. (1992) Natural radioactivity in the aquatic components of the main French rivers, Rad. Prot. Dosim. 45 (1-4), 253-256.

Madelmont C., Rannou A., Renguard H., Jeanmaire L. (1984) Sources externes : cosmique, tellurique et domestique, Congrès sur les données actuelles sur la radioactivité naturelle, Monte-Carlo, 5 7 novembre 1984.

Métivier H., Robé M.C. (1998) Le Radon de l'environnement à l'homme, Coll. IPSN, EDP Sciences, Les Ulis, France.

OPRI (1997) Office de protection contre les rayonnements ionisants, Rapport d'activité 1997 , Le Vesinet, France.

Patti F., Jeanmaire L., Masson M., Pinton Ph., Garcet M. (1990) Temporal variations of iodine-127, potassium-40 and technetium-99 concentrations in Fucus serratus in the English Channel, $J$. Radioanal. Nucl. Chem. 142 (2), 467-480.

Rémy M.L., Lemaître N. (1990) Eaux minérales et radioactivité, Hydrogéol. 4, 267-278.

Rémy M.L., Pellerin P. (1968) Radioactivité naturelle de 250 sources hydrominérales françaises, Bull. INSERM 23 (1), 23-62.

Renoux A. (1984) La radioactivité naturelle de l'air hors des maisons, Congrès sur les données actuelles sur la radioactivité naturelle, Monte-Carlo, 5-7 novembre 1984.

Robé M.C., Rannou A., Le Bronec J., Tymen G. (1992a) Le radon dans les habitations : identification des sources et des voies de transfert et caractérisation des aérosols radioactifs produits, Men. Expl. Cartes géologiques et minières de Belgique, $\mathrm{N}^{\circ} 32$, pp. 93-103.

Robé M.C., Rannou A., Le Bronec J. (1992b) Radon measurement in the environment in France, Rad. Prot. Dosim. 45 (1/4), 455-457.

Ryan T.P., Germain P., Dowdall A.M., Leclerc G., Pollard D. ( 1997) A comparison of ${ }^{210}$ Pu in Mytilus edulis and Fucus vesiculosus in French and Irish Coastal waters. Radioprotection, Colloque radionuclides in the oceans. Radoc 96-97, Proceed. Part 1. Cherbourg-Octeville, France, 7-11 october 1996, Radioprotection 32 (C2), 345-352.

Saito K., Jacob P. (1995) Gamma ray fields in the air due to sources in the ground, Rad. Prot. Dosim. 58 (1), 29-45.

Schmidt S., Reyss J.L., Landre F., Boust D. (1998) Distributions and fluxes of ${ }^{226}$ Ra and ${ }^{228}$ Ra in the Irish sea and in the English Channel, in relation to hydrological conditions and sediment interactions, Rad. Prot. Dosim. 75 (1-4), 65-67.

SFRP (1998) Société française de radioprotection, La radioactivité naturelle en dix épisodes, Journées SFRP, Poitiers, 17-18 mars 1998.

Thébault H., Charmasson S., Calmet D., Arnaud M., Henocque Y. (1998) The Coastal Mediterranean monitoring network project: radionuclide survey, Rad. Prot. Dosim. 75 (1-4), 49-56.

Tort V., Lefaure C., Linden G., Harblet J. (1997) Le tritium dans le milieu aquatique et le risque associé, Radioprotection 32 (4), 501-519.

UNSCEAR (1988) Sources, Effects and Risks of Ionising Radiations, Report to the General Assembly with scientific annexes, E.88.IX.7, Nations Unies, New-York.

UNSCEAR (1993) Sources, Effects and Risks of Ionising Radiations, Report to the General Assembly with scientific annexes, E.94.IX.2, Nations Unies, New-York.

Zettwoog P., Lemaitre N., Bernhard S., Vauzelle Y. (1997) Utilisation de la signature isotopique des radionucléides relâchés par les mines et les usines de traitement de minerais d'uranium pour discriminer aux bas niveaux leur impact environnemental de celui de la radioactivité tellurique naturelle, Radioprotection 32 (4), 467-489 